Applied and

NISTIR 4894

Computational

Mathematics

Division

Computing and Applied Mathematics Laboratory

\title{
Computation of Dendrites Using a Phase Field Model
}

A. A. Wheeler, B. T. Murray, and R. J. Schaefer

$$
\text { July } 1992
$$

Technology Administration

U.S. DEPARTMENT OF COMMERCE

National Institute of Standards and Technology

Gaithersburg, MD 20899 



\section{Computation of Dendrites Using a Phase Field Model}
A. A. Wheeler
B. T. Murray
R. J. Schaefer

U.S. DEPARTMENT OF COMMERCE

Technology Administration

National Institute of Standards

and Technology

Computing and Applied Mathematics Laboratory Applied and Computational Mathematics Division Gaithersburg, MD 20899

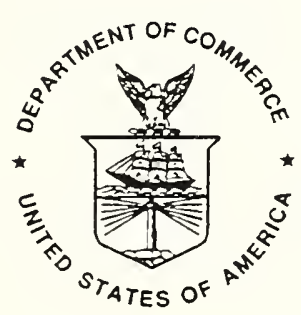

U.S. DEPARTMENT OF COMMERCE

Barbara Hackman Franklin, Secretary

TECHNOLOGY ADMINISTRATION

Robert M. White, Under Secretary for Technology

NATIONAL INSTITUTE OF STANDARDS

AND TECHNOLOGY

John W. Lyons, Director 



\title{
Computation of Dendrites Using
}

\author{
a Phase Field Model
}

\author{
A. A. Wheeler, B. T. Murray and R. J. Schaefer \\ National Institute of Standards and Technology ${ }^{\dagger}$ \\ Gaithersburg, MD 20899 USA
}

\begin{abstract}
A phase field model is used to numerically simulate the solidification of a pure material. We employ it to compute growth into an undercooled liquid for a onedimensional spherically symmetric geometry and a planar two-dimensional rectangular region. The phase field model equations are solved using finite difference techniques on a uniform mesh. For the growth of a sphere, the solutions from the phase field equations for sufficiently small interface widths are in good agreement with a numerical solution to the classical sharp interface model obtained using a Green's function approach. In two dimensions, we simulate dendritic growth of nickel with four-fold anisotropy and investigate the effect of the level of anisotropy on the growth of a dendrite. The quantitative behavior of the phase field model is evaluated for varying interface thickness and spatial and temporal resolution. We find quantitatively that the results depend on the interface thickness and with the simple numerical scheme employed it is not practical to do computations with an interface that is sufficiently thin for the numerical solution to accurately represent a sharp interface model. However, even with a relatively thick interface the results from the phase field model show many of the features of dendritic growth and they are in surprisingly good quantitative agreement with the Ivantsov solution and microscopic solvability theory.
\end{abstract}

*Permanent address: School of Mathematics, University of Bristol, Bristol BS8 1TW, U.K.

${ }^{\dagger}$ Technology Administration, U.S. Department of Commerce, Washington D.C. 


\section{Introduction}

Recently Kobayashi [1], [2], [3] reported computations of the unsteady phase field equations in two and three spatial dimensions, which clearly showed the evolution of solid dendritic structures into an undercooled melt. Other realistic features of dendritic growth were also exhibited in these computations, such as tertiary side arm formation, side arm coarsening well away from the dendrite tip, and the inclusion of liquid droplets in the solid phase. This, to our knowledge, is the first time that a computation of solidification has been able to show dendritic growth with the above features. Kobayashi's work is a qualitative demonstration of the possible utility of phase field models of solidification as a computational tool for modeling complicated, realistic solid/liquid interfaces. However, he did not systematically investigate such important issues as the accuracy of his numerical solutions, their relation to the various classical formulations of solidification, such as the Stefan problem, or whether his simulations were conducted in a parameter regime that corresponded to realistic growth conditions of an actual material.

It is the focus of this paper to address these issues, and critically assess phase field models as a viable computational technique. We go on to compare the results of our computations of dendritic growth using a phase field model with current theories of dendrite tip selection, in particular, the Ivantsov solution, marginal stability theory, and microscopic solvability theory. We also address the issue of side arm formation. In contrast to Kobayashi, our work represents a quantitative evaluation of phase field models, as well as the theories of dendrite tip selection mentioned above.

Phase field models of solidification, since their invention by Langer, see [4], following an adaptation of the Model $\mathrm{C}$ proposed by Halperin et al. [5], and also independently by Collins and Levine [6], have been subject to development and rigorous mathematical analysis by Caginalp [7], [8]. Only recently has computer technology advanced sufficiently that it is now possible to numerically integrate the unsteady phase field equations, in realistic configurations. An early computation is due to Smith [9], and more recently accurate calculations in one spatial dimension have been obtained by Caginalp and Socolovsky [10] 
and Lowen et al. [11]. The advantage of the phase field formulation of solidification, which is described in section 2 , is that no distinction is made between the solid, liquid and interface. This allows the whole domain to be treated in the same way numerically; the interface is not tracked but given implicitly by the level set of a scalar function of time and space, the so-called phase field. In essence, the classical formulation of a free boundary problem is replaced by a pair of nonlinear reaction-diffusion equations for the temperature and phase field. This approach allows the computation of realistic complicated interfacial structures whose connectedness changes in time. Numerical treatments of free boundary problems, using boundary integral or domain transformation methods encounter great difficulties in these situations.

Experiments on the free growth of dendrites by, for example, Glicksman et al. [12], show that the dendrite tip selects an operating state, as characterized by the tip velocity, $v$, and radius of curvature, $r$, dependent on the undercooling of the melt, $\Delta T$. Ivantsov's theory [13] provides a local model of a dendrite tip, which is represented by a parabola, solidifying with constant velocity into an infinite melt. This theory determines the Peclet number, $\mathcal{P}=2 v r / \kappa$, as a function of the undercooling parameter, $\Delta=c \Delta T / L$, where $c$ is the specific heat, $\kappa$ is the thermal diffusivity, and $L$ is the latent heat per unit volume. Another independent relation between $v$ and $r$ is required to determine them uniquely. A resolution of this indeterminacy was sought by introducing surface energy into the theory, which introduces an additional length scale, $d_{0}=\sigma / L$, the capillary length into the problem, where $\sigma$ is the surface energy. This approach resulted in marginal stability theory, originally due to Oldfield [14], which predicts the other relation to be $\sigma^{\star}\left(=2 \kappa d_{0} / v r^{2}\right)=$ 0.0192. This theory is ad hoc and based on the notion that the tip exists in a state of marginal stability related to the critical radius for growth of a solid sphere or cylinder into an undercooled melt.

In contrast, an alternate mathematical treatment incorporating the surface energy is known as microscopic solvability theory. It is reviewed by Kessler et al. [15] and Pomeau and Ben Amar [16]. This theory indicates that surface energy in itself is not sufficient, but that anisotropy of surface energy is required to achieve a steady stable tip, and $\sigma^{*}=$ 
$\mathcal{O}\left(\gamma^{7 / 4}\right)$ as $\gamma \rightarrow 0$, where $\gamma$ is the surface tension anisotropy parameter, defined in twodimensions by $\sigma \propto 1+\gamma \cos (k \theta)$, where $\theta$ is the angle of the interface to a given direction and $k$ is an integer that characterizes the symmetry of the crystal. Unfortunately, the physical basis for this is unclear, the theory relying on sophisticated formal asymptotic methods. To date, to our knowledge, there is scant independent confirmation of microscopic solvability theory against numerical simulation or experiments. Meiron [17] showed from a numerical boundary integral formulation, that anisotropy is necessary for stable, steady growth of the tip of a needle crystal. Saito et al. [18] conducted a numerical simulation on a quasisteady model using a Greens function technique, and were able to roughly confirm the predictions of the theory. Experimental confirmation, the ultimate test of such a theory, is much less conclusive. This is because the anisotropy in the surface energy of most materials is difficult to measure and thus unknown. Also microscopic solvability theory is most well-developed for two dimensions, but experimentally dendrites are usually three dimensional. Ben Amar [19] has shown that by choosing the best value of the anisotropy parameter the experimental results of Willnecker et al. [20] could be adequately described over a wide range of values of the dimensionless undercooling parameter. However, careful experiments on pivalic acid, camphene and succinonitrile by Rubinstein and Glicksman [21], [22] do not support microscopic solvability theory, in particular, they do not confirm the dependence $\sigma^{\star}=\mathcal{O}\left(\gamma^{7 / 4}\right)$ as $\gamma \rightarrow 0$.

In section 2 we briefly introduce a new phase field model for solidification based on an entropy functional formulation. In section 3 we describe the results of our numerical integration of the phase field equations in a spherically symmetric geometry, and quantitatively compare our results to an accurate numerical solution of the corresponding free-boundary problem based on a Greens function technique due to Schaefer and Glicksman [23]. In section 4, we describe the numerical method used to solve the unsteady phase field equations in a planar two dimensional geometry and make a quantitative assessment of it. We compare our results to both microscopic solvability theory and marginal stability theory, as well as discuss side-arm formation.

Our results in the case of the spherically symmetric geometry indicate that the nu- 
merical solution of the phase field equations converge to the results of the corresponding free-boundary problern as the interface thickness is reduced providing that the interface is adequately resolved. In two-dimensional planar geometry, the validation of the numerical solution of the phase field equations is much more difficult to perform. This is because adequate resolution of the interfacial layer in a realistic parameter range is difficult to achieve. Qualitatively, our numerical solutions show dendrite formation for growth into an undercooled melt, and show the importance of the level of surface tension anisotropy. We find reasonable quantitative agreement to the Ivantsov solution and to microscopic solvability theory for a fixed value of the interface thickness; however, we show that the results are dependent on the interface thickness. This, we believe, provides the main obstacle to the use of phase field methods for the accurate computation of complex solid/liquid interfaces for realistic growth configurations in more than one space dimension. Nevertheless, the phase field method produces many of the qualitative features observed in real crystal growth, and with further development of the numerical solution techniques may provide the best approach for simulating dendritic growth.

\section{The Governing Equations}

Phase field models of the solidification of a pure material are based on a Landau-Ginzberg free-energy functional:

$$
\mathcal{F}=\int_{\Omega}\left[f(\phi, T)+\frac{\epsilon^{2}}{2}(\nabla \phi)^{2}\right] d \Omega,
$$

where $\Omega$ is the region occupied by the system, $\phi(\mathbf{x}, t)$ is the phase-field, $T(\mathbf{x}, t)$ is the temperature and $\epsilon$ is a parameter which is constant for an isotropic material. The freeenergy density $f(\phi, T)$ is a double well with respect to $\phi$. Various choices for the precise form of $f$ have been suggested, the most studied of which is

$$
f(\phi, T)=\frac{1}{8 a}\left(1-\phi^{2}\right)^{2}-\left(T-T_{M}\right) \phi,
$$

where $a$ is a positive constant, and $T_{M}$ is the melting temperature of the material. The solid and liquid phases are represented by $\phi$ in the neighborhood of -1 and +1 respectively. 
An alternative choice for $f$ has been proposed by Kobayashi [2]:

$$
f(\phi, T)=W \int_{0}^{\phi} \zeta(\zeta-1)\left(\zeta-\frac{1}{2}-\beta\left[T-T_{M}\right]\right) d \zeta,
$$

where $W$ is a constant and $\beta$ is a monotonic function of $T-T_{M}$, with $|\beta|<1 / 2$ and $\beta(0)=0$. This choice has the advantage over Eq. (2) that the liquid and solid states are represented by $\phi$ exactly equal to zero and unity respectively.

In both cases the free energy Eq. (1) is used to derive a kinetic equation for the phase field by requiring that it evolves in a manner such that total free energy $\mathcal{F}$ decreases monotonically in time. The simplest choice is made consistent with this requirement:

$$
\phi_{t} \propto-\frac{\delta \mathcal{F}}{\delta \phi} .
$$

To this is appended the heat equation modified to take account of the liberation of latent heat, by the inclusion of an appropriate source term:

$$
\frac{\partial T}{\partial t}+K \frac{\partial \phi}{\partial t}=\nabla^{2} T
$$

which provides an equation for the temperature, where $K$ is a constant proportional to the latent heat per unit volume. Typically, Eq. (5) is not derived from basic thermodynamic principles with specific consideration of the form of the free-energy functional Eq. (1), so it is not clear that the solution of the equations Eq. (4) and Eq. (5) will ensure that $\mathcal{F}$ is monotonic decreasing in time. Penrose and Fife [24] have addressed this question by employing the appropriate thermodynamic potential to this non-isothermal situation, namely an entropy functional. From this they derived, in a consistent manner, the phase field equations when the free energy density is given by Eq. (2). To our knowledge, the phase field model based on the free energy density given by Eq. (3) has not been placed in a consistent thermodynamic setting in the same way. However, this free energy has the advantage that, because the two states, solid and liquid, are given by fixed values of $\phi$, 0 and 1, the latent heat released through the source term in the modified heat Eq. (5) is correctly accounted for in a numerical computation of the phase field equations given by Eq. (4) and Eq. (5). This is not true with the choice for $f$ given by Eq. (2) as the values of $\phi$ representing the solid and liquid states depend upon $\epsilon, T$ and $a$. 
Another phase field model which combines the advantages of both the above models, that is, one that is thermodynamically consistent and represents the solid by $\phi=0$ and the liquid by $\phi=1$, has been suggested and is discussed at length in [25]. This new model results in the following dimensionless governing equations for the phase field and temperature:

$$
\begin{gathered}
\frac{\tilde{\epsilon}^{2}}{m} \frac{\partial \phi}{\partial \tau}=\phi(1-\phi)\left[\phi-\frac{1}{2}+30 \tilde{\epsilon} \alpha \Delta u \phi(1-\phi)\right]+\tilde{\epsilon}^{2} \nabla^{2} \phi, \\
\frac{\partial u}{\partial \tau}+\frac{1}{\Delta} p^{\prime}(\phi) \frac{\partial \phi}{\partial \tau}=\nabla^{2} u,
\end{gathered}
$$

where $p(\phi)=\phi^{3}\left(10-15 \phi+6 \phi^{2}\right)$ and prime denotes differentiation. The solution of these model equations results in the total dimensionless entropy of the system $\mathcal{S}$, given by

$$
\left.\mathcal{S}=\int_{\Omega}\left[s(\phi, u)-\frac{\tilde{\epsilon}^{2}}{2}(\nabla \phi)^{2}\right)\right] d \Omega
$$

increasing monotonically in time, with the dimensionless entropy density given by

$$
s(\phi, u)=\int_{0}^{\phi}\left[\zeta(1-\zeta)\left(\zeta-\frac{1}{2}\right)+\tilde{\epsilon} \alpha \Delta u p^{\prime}(\zeta)\right] d \zeta .
$$

Here, length has been scaled on some reference length scale $w$ of say the dimensions of the domain, time on the corresponding thermal diffusion time $w^{2} / \kappa$, where $\kappa$ is the thermal diffusivity, and temperature by putting $T=T_{M}+\Delta T u$, where $\Delta T$ is a reference temperature difference (such as, between the melting temperature and the temperature at the boundary of the domain).

The present phase field model is characterized by four dimensionless parameters. The parameter $\Delta$ is the dimensionless undercooling, defined as

$$
\Delta=\frac{c \Delta T}{L}
$$

where $c$ is the specific heat and $L$ is the latent heat per unit volume. In [25], it is shown how the remaining three constants are related to the physical parameters which characterize the interface dynamics (i.e., interfacial energy, $\sigma$, and mobility, $\mu$ ) and to an estimate of the interface thickness, $\delta$, which is a consequence of the phase field approach; the following definitions relate the remaining model parameters to the physical constants:

$$
\alpha=\frac{\sqrt{2} w L^{2}}{12 c \sigma T_{M}}
$$




$$
m=\frac{\mu \sigma T_{M}}{\kappa L}
$$

and

$$
\tilde{\epsilon}=\frac{\delta}{w}
$$

Once the characteristic length scale $w$ has been chosen, knowledge of the physical properties leaves one degree of freedom, namely $\tilde{\epsilon}$, which then is used to set the interface thickness. It is expected that in order to model the physical behavior correctly, the interface thickness must be sufficiently small compared to the interfacial macrostructures that we wish to model; however, from a computational viewpoint, it is desirable for the interface thickness to be as large as possible in order that accurate solution of the phase field equations can be obtained for practical computational effort. This is one of the key issues to be addressed by the computations presented here.

From Eq. (13) it is clear that the limit $\tilde{\epsilon} \rightarrow 0$ corresponds to the interfacial thickness tending to zero. Specifically, in [25] we show that if we take the limit $\tilde{\epsilon} \rightarrow 0$, where the constants $\alpha$ and $m$ defined above are assumed to be order one, we recover the sharp interface model to leading order

$$
\frac{\partial u}{\partial \tau}=\nabla^{2} u
$$

with interfacial boundary conditions

$$
\begin{aligned}
& \left.\frac{\partial u}{\partial n}\right|_{\text {solid }} ^{\text {liquid }}=-\frac{1}{\Delta} v_{n}, \\
& u=-\Gamma\left(\frac{v_{n}}{m}+\mathcal{K}\right),
\end{aligned}
$$

where $\Gamma=\sigma T_{m} /(w L \Delta T)$ is the dimensionless capillary length, $v_{n}$ is the dimensionless normal velocity of the interface (into the liquid) and $\mathcal{K}$ is the curvature. The conservation of heat across the solid-liquid interface allowing for latent heat production is represented by Eq. (15) and the Gibbs-Thomson equation modified to account for interface kinetics by Eq. (16). 


\section{The Growth Of A Sphere}

Our first test of the phase field model is to compare it to an accurate numerical solution of the growth of a sphere into an undercooled melt originally described in Schaefer and Glicksman [23]. The growth of the sphere is strongly influenced by curvature, kinetic, and heat flow effects, thus providing the ingredients for a severe test of the correspondence between sharp interface and phase field descriptions in the limit $\tilde{\epsilon} \rightarrow 0$.

Schaefer and Glicksman [23] solved the sharp interface problem Eq. (14), Eq. (15), and Eq. (16), recovered in the limit $\tilde{\epsilon} \rightarrow 0$ in a spherically symmetric geometry using a Greens function method. We have used the same technique here modifying slightly their normalization to be consistent with that we use here in the phase field model. The initial data corresponded to a solid sphere of radius $\rho_{0}$ chosen to be $10 \%$ greater than the equilibrium radius $\rho^{*}$. In our non-dimensionalization, we choose $\rho^{*}$ as the reference length scale $w$, and $\Delta T$ to be equal to the undercooling (taken to be positive). Thus, initially we set $u=-1$ everywhere. The dimensionless equilibrium radius is given from Eq. (16) as $\rho^{*}=1 /(3 \sqrt{2} \alpha \Delta)$, and because we choose $\rho^{*}=1$ this gives $\alpha=1 /(3 \sqrt{2} \Delta)$. Also, we introduce the parameter

$$
\xi=\frac{\mu \sigma T_{M}}{2 \kappa L}
$$

which represents the mobility of the interface and is the same dimensionless group employed in [23], and we note that $m=2 \xi$. The nondimensional boundary condition for the interfacial temperature Eq. (16) is given in terms of $\xi$ as

$$
\frac{d \rho}{d \tau}=-4 \xi\left(u+\frac{1}{\rho}\right)
$$

\subsection{Numerical Method For Solution Of the Sharp Interface Problem}

As the sphere grows, heat is emitted from its surface at a rate proportional to the latent heat and to the growth rate. The resulting temperature field, and in particular the surface temperature of the growing sphere at $r=\rho(\tau)$, is calculated by a Green's function integral:

$$
u(\rho, \tau)=-1+\int_{0}^{\tau} G\left(\rho, \rho^{\prime}\left(\tau^{\prime}\right), \tau, \tau^{\prime}\right) \frac{d \rho^{\prime}}{d \tau^{\prime}} d \tau^{\prime}
$$


where for a sphere

$$
G\left(r, r^{\prime}, \tau, \tau^{\prime}\right)=\frac{r^{\prime}}{2 r \sqrt{\pi\left(\tau-\tau^{\prime}\right)}}\left[\exp \left(-\frac{\left(r-r^{\prime}\right)^{2}}{16\left(\tau-\tau^{\prime}\right)^{2}}\right)-\exp \left(-\frac{\left(r+r^{\prime}\right)^{2}}{16\left(\tau-\tau^{\prime}\right)^{2}}\right)\right]
$$

The radius of the sphere is given simply by

$$
\rho(\tau)=\rho_{\circ}+\int_{0}^{\tau} \frac{d \rho^{\prime}}{d \tau^{\prime}} d \tau^{\prime}
$$

The numerical procedure used to increment the interface position $\rho_{n}$ and temperature $u_{n}$ at the nth time step $\left(\tau=\tau_{n}=n \Delta \tau\right)$ to the $\mathrm{n}+1$ th time $\operatorname{step}\left(\tau_{n+1}=\tau_{n}+\Delta \tau\right)$ was as follows:

1. Compute $d \rho / d \tau$ at $\tau=\tau_{n}$, denoted by $\dot{\rho}_{n}$, from Eq. (18).

2. Compute $\rho_{n+1}$ from Eq. (21) employing a backward difference, i.e., $\rho_{n+1}=\rho_{n}+\dot{\rho}_{n} \Delta \tau$.

3. Update the interfacial temperature from Eq. (19) by

$$
u\left(\rho_{n}, n \Delta \tau\right)=\Delta \tau \sum_{i=1}^{i=n} \dot{\rho}_{i-1} G\left(\rho_{n}, \rho_{i}, n \Delta \tau, i \Delta \tau\right)
$$

An alternative quadrature formula of the Greens function integral in step 3 was also tried using only $\rho_{i-1}$ on each time interval and was found to converge much less rapidly. For the conditions considered in this paper, calculations were carried out using a range of values of the time step. It was found that for time steps smaller than a value, which depends on $\Delta$ and $\xi$, the results agreed within $0.1 \%$.

\subsection{Numerical Method For The Phase Field Model}

We assume that initially the temperature is everywhere uniform at $u \equiv-1$. We employed a finite difference scheme to solve the phase field equations Eq. (6) and Eq. (7) expressed in a spherically symmetric geometry in which the sole spatial variable is $r$, the radial distance. Second-order central differences were used to discretize the Laplacian operator on a uniform spatial mesh for the domain $0 \leq r \leq R$, with $N+1$ nodes. Unlike the Greens function approach described above the phase field computation requires a finite domain 
and thus we must be careful in choosing appropriate outer boundary conditions at $r=R$. We chose them to be

$$
\frac{\partial u}{\partial r}+\frac{u+1}{R}=0, \text { and } \frac{\partial \phi}{\partial r}=0 \text { at } r=R
$$

which is consistent with assuming a quasi-static approximation for the heat diffusion equation in the far field. The phase field is simply equal to unity in the far field. At the origin the coordinate singularity in the Laplacian operator was avoided by employing a local form for the solution as described in Smith [9] and Neumann boundary conditions. The solution was advanced in time using a second-order Crank-Nicholson scheme for the Laplacian terms and a backward difference for the latent heat production term in the modified heat equation. Specifically, the solution was advanced a single time step in the following manner:

1. Update the phase field by solving the tridiagonal system,

$$
\mathcal{T}_{\phi}^{+}\left(\Phi^{n+1}\right)=\mathcal{T}_{\phi}\left(\Phi^{n}\right)+\mathbf{s}\left(\Phi_{n}, \tilde{U}^{n}\right)
$$

2. Update the temperature by solving the tridiagonal system,

$$
\mathcal{T}_{u}^{+}\left(\tilde{\mathbf{U}}^{n+1}\right)=\mathcal{T}_{u}\left(\tilde{\mathbf{U}}^{n}\right)-\mathrm{l}\left(\Phi^{n}\right)
$$

Here $\Phi^{n}$ and $\tilde{U}^{n+1}$ are the discrete approximations to $\phi$ and $u, \mathcal{T}_{\phi}^{+}$etc represent the tridiagonal matrices that result from the temporal-spatial discretization, $\mathbf{s}_{i}\left(\Phi_{n}\right)=s_{\phi}\left(\Phi_{i}^{n}, \tilde{U}_{i}^{n}\right)$ and $l_{i}\left(\Phi^{n}\right)=l\left(\Phi_{i}^{n+1}\right)-l\left(\Phi_{i}^{n}\right)$. The computations were conducted on a Cray-YMP and the solution of the tridiagonal systems was conducted using the Cray library routine TRID which employs cyclic reduction. The code was well vectorized and achieved in excess of 200 MFLOPS on a single processor.

\subsection{Comparison of the Two Methods}

We conducted calculations using both methods for an undercooling of 0.5 and two different values of the mobility, $\xi=0.05$ and 1.0. The most comprehensive set of computations 
was conducted for $\xi=1.0$. For this case in conducting the computations on the phase field model, we used five different values of $\tilde{\epsilon}(0.1,0.075,0.05,0.02$ and 0.01$)$ and five different grids with mesh spacings $\Delta r(0.001,0.002,0.01,0.02$ and 0.1$)$ for each value of $\tilde{\epsilon}$. The time step was taken to be $10^{-4}$. It was found that there was a critical value of the time step, that was apparently insensitive to the mesh size, above which the numerical scheme was unstable, despite the implicit discretization of the diffusion operator. We ascribe this to the explicit discretization of the source terms in the phase field and heat equation. It was found that invoking the outer boundary condition at $r=10$ was sufficient for approximating an unbounded domain.

For the case $\xi=0.05$, which is a more realistic value for a supercooled pure metal, calculations were only performed on the phase field model for $\tilde{\epsilon}=0.01$, with $\Delta r=0.05$, and $\Delta \tau=10^{-3}$, for which we were able to obtain excellent agreement with the numerically computed solution of the classical formulation, but with the outer boundary placed further away at $r=20$. We attribute this to the increased communication by the temperature

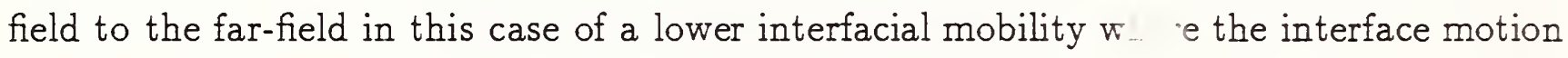
is relatively sluggish relative to the thermal diffusion time. Herceforth, we restrict our discussion to the case $\xi=1.0$.

On the finest grids excellent agreement was obtained between the two procedures as shown in Figure 1 for $\Delta r=0.001$ and $\tilde{\epsilon}=0.01$. It was found that the numerical solution exhibited a temporal oscillation when the mesh was too coarse. In all except one of these cases the breakdown corresponded to values of $\tilde{\epsilon} / \Delta r$ (a measure of the resolution of the interface by the computational mesh) less than or equal to unity. In particular, for a fixed value of $\tilde{\epsilon}$, on increasing the mesh spacing the numerical instability first manifested itself as a significant oscillation in the interface velocity; at larger values of $\Delta r$ it was apparent as a significant oscillation in the surface temperature also. These observations broadly confirm the suggestion by Osher [26] and Caginalp and Socolovsky [10] that the numerical solution of the phase field equations by a finite difference method on a uniform mesh could be expected to breakdown for $\Delta r>\tilde{\epsilon}$.

We now discuss the solutions computed on sufficiently fine meshes for which the numer- 


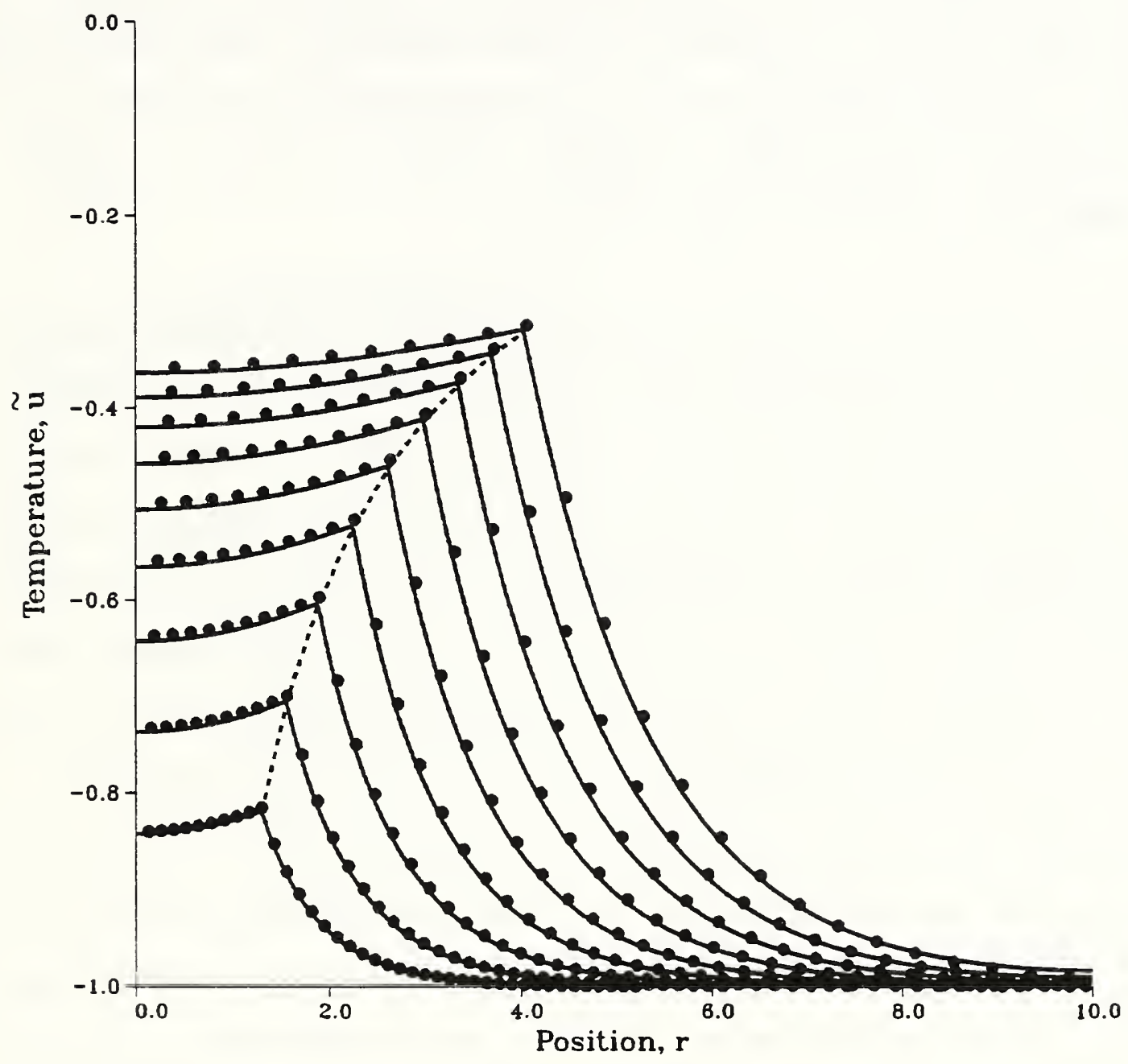

Figure 1: The solid curves (from bottom to top) are the temperature at times $\tau=1.25$, $2.5,3.75, \ldots, 11.25$, as a function of the radial coordinate, computed from the phase field model for the case $\tilde{\epsilon}=0.01, \Delta=0.5$ and $\xi=1.0$. The dashed curve represents the locus of the interface $\phi=0.5$ and the almost coincident solid circles are taken from the Greens function solution at the corresponding time levels. 


\begin{tabular}{||c||l|l|l|l|l||}
\hline \hline$\Delta r$ & $\tilde{\epsilon}=0.01$ & $\tilde{\epsilon}=0.02$ & $\tilde{\epsilon}=0.05$ & $\tilde{\epsilon}=0.075$ & $\tilde{\epsilon}=0.1$ \\
\hline 0.001 & 1.520 & -0.7502 & -3.347 & -5.213 & -7.058 \\
0.002 & 1.504 & -0.7540 & -3.347 & -5.213 & -7.058 \\
0.01 & 1.009 & -0.8753 & -3.361 & $\mathbf{- 5 . 2 1 7 3}$ & -7.060 \\
0.02 & 92.44 & -1.2452 & -3.404 & -5.2300 & -7.063 \\
0.1 & 91.49 & 9.149 & -3.086 & -5.6091 & -7.063 \\
\hline \hline
\end{tabular}

Table 1: The relative difference in the surface temperature calculated by the two methods over an interval of 12 time units expressed as a percentage. The entries in italics indicate that the solution was subject to a numerical oscillatory instability. Those in bold were employed in the curve fitting discussed in section 3.3; in particular, the emboldened entries in the columns were fitted as $-0.748337-0.00153 s-0.52 s^{2}$, for $\tilde{\epsilon}=0.02 ;-3.34638-$ $0.000375 s-0.36 s^{2}$, for $\tilde{\epsilon}=0.05 ; 5.21304-0.00462 s-0.22 s^{2}$, for $\tilde{\epsilon}=0.075 ;-7.05878-$ $0.0330495 s+0.284232 s^{2}$, for $\tilde{\epsilon}=0.1$, where $s=\Delta r / \tilde{\epsilon}$.

ical oscillation was not present. We found the best agreement in the interface temperature between the two methods was obtained when it was expressed as a function of the interface position, the disparity being insensitive to $\tilde{\epsilon} / \Delta r$ (in the phase field formulation the interface position was defined to be given by $\phi=1 / 2$ ). The interface temperature as a function of interface position is controlled largely by heat flow, and this excellent agreement indicates that our scheme provides a good solution of the heat equation. However, the agreement between the two methods for the interface temperature $u_{i}$ (as well as its position $\rho$ and velocity $\dot{\rho}$ ), expressed as a function of time depended much more strongly on the values $\tilde{\epsilon}$ and $\Delta r$. In particular, the disparity between the two numerical methods increased monotonically with time. This distinction between the errors in the interface temperature expressed as a function of time or position is illustrated in Figure 2 for $\tilde{\epsilon}=0.1$. The central dashed line indicates the interface temperature as a function of position, as computed by the phase field method. This curve is almost coincident with the solid circles which represent the same quantity but obtained from the Greens function method. However, each solid circle is not coincident with the interface temperature for the corresponding time level, thus indicating a considerable disparity in the two methods when the interfacial temperatures are compared as a function of time. 


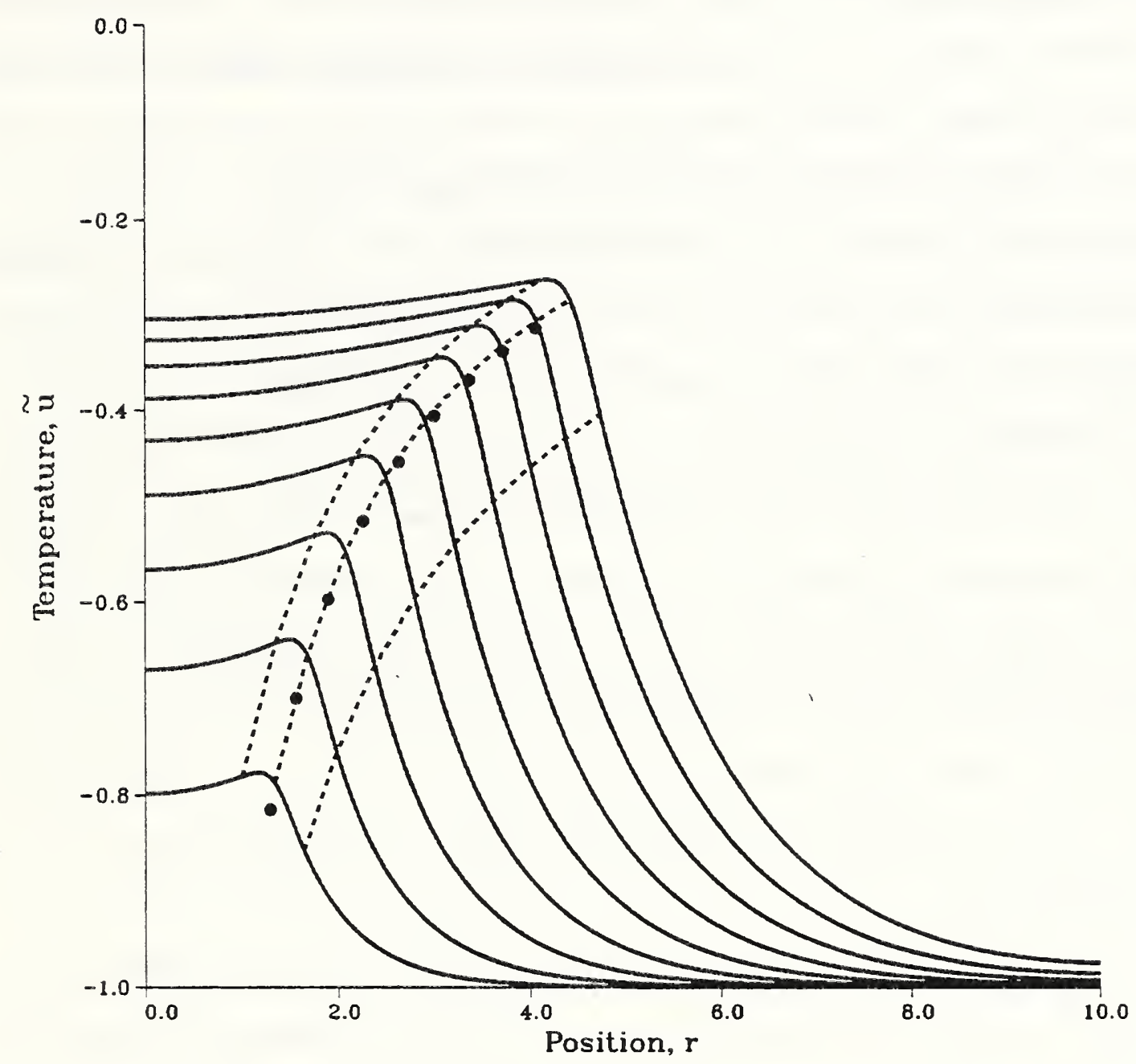

Figure 2: The solid curves (from bottom to top) are the temperature at times $\tau=1.25$, $2.5,3.75, \ldots, 11.25$, as a function of the radial coordinate, computed from the phase field model for the case $\tilde{\epsilon}=0.1, \Delta=0.5$ and $\xi=1.0$. The dashed curves are the locus of (from left to right) $\phi=0.1, \phi=0.5$ and $\phi=0.9$. The solid circles represent the locus of interface temperatures computed from the Greens function solution; each solid circle corresponds to the same time levels as the solid curves, and the time levels increase upwards. 
A summary of the errors between the two methods is given Table 1, where the error between the two techniques in the interface temperature (averaged as a function of time over the computational time interval of 12 time units) is tabulated in terms of the mesh spacing and $\tilde{\epsilon}$. The italicized entries indicate an unstable numerical solution, as discussed above. The variation in the errors of $\rho$ and $\dot{\rho}$ showed similar behavior to the interface temperature. For a fixed value of the mesh spacing the error between the two techniques decreases as $\tilde{\epsilon}$ decreases, except for the case of reduction of $\tilde{\epsilon}$ from $2 \times 10^{-2}$ to the smallest value $10^{-2}$. This we attribute to the error between the exact phase field solution and the classical solution being sufficiently small for $\tilde{\epsilon}=0.01$ that it is comparable to the numerical errors in the Greens function solution of the classical problem, as well as the errors in the quadrature formula (with a time step of 0.05 ) used to compute the average surface temperature from the numerically computed phase field solution. Linear regression of the errors against $\tilde{\epsilon}$ for fixed values of $\Delta r$ show the error between the numerical solution of two different formulations to be first order, i.e., $\propto \lambda \tilde{\epsilon}$, where the constant $\lambda$ was found to be $78.6,78.56,77.13,73.32$ for $\Delta r=0.001,0.002,0.01,0.02$, respectively. The convergence with $\tilde{\epsilon}$ is therefore insensitive to the mesh size (assuming that numerical instability is not present). It also confirms the asymptotic theory of the phase field equations in the limit $\tilde{\epsilon} \rightarrow 0$ by Caginalp [8] which indicates that their solution approaches the sharp interface solution in this limit. This strong linear dependence of the error on $\tilde{\epsilon}$ for a fixed mesh allows the application of accelerated convergence techniques, such as Richardsons method, to the phase-field equations.

With the value of $\tilde{\epsilon}$ fixed the errors decreased as the mesh spacing was refined, albeit very slowly. There was one exception to this for $\tilde{\epsilon}=0.01$, where regression of the errors with respect to $\Delta r$ indicates that this is not so. This we attribute, as discussed above, to comparable errors associated with the Greens function technique and the quadrature formula used when $\tilde{\epsilon}$ is sufficiently small. We fitted a quadratic polynomial to the errors as a function of $s=\Delta r / \tilde{\epsilon}$ for $\tilde{\epsilon}=0.02,0.05,0.075$ and 1.0. The values of the coefficients are given in Table 1 and show that the convergence is predominantly quadratic; however, the coefficients do depend on $\tilde{\epsilon}$. 
In summary, these calculations reveal that a numerical instability occurs when either the time step exceeds a critical value, which is independent of the mesh size, or the mesh spacing is approximately greater than or equal to $\tilde{\epsilon}$. The optimum value of $\Delta r$ is given by $\Delta r \approx \tilde{\epsilon}$ because the error is not significantly reduced by using a finer mesh compared to the consequent increase in processor time. The errors themselves appear to be $\mathcal{O}(\tilde{\epsilon})$ and dependent only weakly on the mesh spacing provided $\Delta r \leq \tilde{\epsilon}$.

\section{Two-Dimensional Calculations}

In addition to the spherically symmetric calculations previously described, a series of twodimensional numerical calculations are performed in order to further evaluate the present phase field model. The primary objective is to evaluate the behavior of the given phase field model for realistic physical parameter values, i.e., evaluate how well the diffuse interface model reproduces the essential physical mechanisms, and investigate the feasibility of accurate numerical simulations of unconstrained solidification of a pure material in an undercooled melt.

For the two-dimensional calculations, the phase field equation given by Eq. (6) is modified to include anisotropy in the parameter $\tilde{\epsilon}$, where the anisotropy is assumed to have the form

$$
\tilde{\epsilon}(\theta)=\bar{\epsilon} \eta(\theta)=\bar{\epsilon}(1+\gamma \cos k \theta) .
$$

The angle $\theta$ is defined as the angle between the normal to the interface and the $x$-axis, and $k$ specifies the mode number. Evaluating the variational derivative of Eq. (1) after including the variation of $\tilde{\epsilon}$ with orientation yields a modified version of Eq. (6):

$$
\begin{aligned}
& \frac{\bar{\epsilon}^{2}}{m} \frac{\partial \phi}{\partial \tau}=\left\{\phi(1-\phi)\left[\phi-\frac{1}{2}+30 \bar{\epsilon} \alpha \Delta u \phi(1-\phi)\right]-\bar{\epsilon}^{2} \frac{\partial}{\partial x}\left(\eta(\theta) \eta^{\prime}(\theta) \frac{\partial \phi}{\partial y}\right)\right. \\
&\left.+\bar{\epsilon}^{2} \frac{\partial}{\partial y}\left(\eta(\theta) \eta^{\prime}(\theta) \frac{\partial \phi}{\partial x}\right)+\bar{\epsilon}^{2} \nabla \cdot\left(\eta^{2}(\theta) \nabla \phi\right)\right\} .
\end{aligned}
$$

In the phase field model, the interface is represented by level sets of $\phi$. In order to compute the anisotropic behavior, the orientation angle is determined in terms of the phase field $\phi$ 
using the following relation for the normal vector:

$$
\hat{n}=\frac{\nabla \phi}{|\nabla \phi|}=\cos \theta \hat{x}+\sin \theta \hat{y} .
$$

From this expression we have the definition

$$
\tan \theta=\frac{\phi_{y}}{\phi_{x}}
$$

and, in addition, we obtain

$$
\theta_{x}=\frac{\phi_{x} \phi_{x y}-\phi_{y} \phi_{x x}}{|\nabla \phi|^{2}}, \quad \theta_{y}=\frac{\phi_{x} \phi_{y y}-\phi_{y} \phi_{x y}}{|\nabla \phi|^{2}} .
$$

These relations are used to compute the terms in Eq. (23) which arise from expanding the derivatives of $\tilde{\epsilon}(\theta)$.

It is shown in [27] that the only modification to the free boundary problem Eq. (14) to Eq. (16), obtained in the limit $\tilde{\epsilon} \rightarrow 0$, is to the modified Gibbs-Thomson equation which becomes

$$
u=-\Gamma\left\{\frac{v_{n}}{m[\eta(\theta)]^{2}}+\left[\eta(\theta)+\eta^{\prime \prime}(\theta)\right] \mathcal{K}\right\} .
$$

We note that allowing $\tilde{\epsilon}$ to depend on $\theta$ modifies both the interface kinetic and curvature terms. It is shown in [27] that the latter is the same as that obtained from sharp interface models where the surface energy depends on the interface orientation.

For the two-dimensional simulations, symmetry conditions (vanishing Neumann conditions for both $\phi$ and $u$ ) are applied at the boundaries of the rectangular domain, which has scaled dimensions of $X_{L}$ and $Y_{L}$ in the $x$ and $y$ coordinate directions, respectively. We note that the results presented here for the temperature and phase field are displayed after reflecting the computational domain about the line $y=0$, which corresponds to the axis of the dendrite. From the chosen definition of the dimensionless variables, the value $u=0$ corresponds to the melting temperature of the pure material, while $u=-1$ is the undercooling temperature (the dimensional undercooling is $\Delta T$ ). Initially for each calculation, a small region of solid $(u=0)$ is located at the $x=0, y=0$ corner of the domain, and the remainder of the domain is undercooled liquid $(u=-1)$. The shape of the initial 
solid region is either one-quarter of a circle with an initial radius denoted by $r_{0}$ or onequarter of an ellipse with semi-minor axis $y_{0}$ and semi-major axis $x_{0}$. Either continuous initial conditions, for which the values of $\phi$ and $u$ change smoothly over a thin region, or discontinuous conditions were used in the computations. For the results presented, the long-time behavior of the solutions is insensitive to the type of initial conditions assumed.

The two-dimensional simulations were performed using property values for pure nickel. The values for the required material parameters are: $\sigma=3.7 \times 10^{-5} \mathrm{~J} / \mathrm{cm}^{2}, T_{m}=1728 \mathrm{~K}$, $L=2350 \mathrm{~J} / \mathrm{cm}^{3}, c=5.42 \mathrm{~J} / \mathrm{K} \mathrm{cm} \mathrm{cm}^{3}, \kappa=0.155 \mathrm{~cm}^{2} / \mathrm{s}$, and $\mu=285 \mathrm{~cm} / \mathrm{K} \mathrm{s}$. Except for the kinetic coefficient, $\mu$, these property values for nickel are readily available (see [28]). The value for the kinetic coefficient lies in the range of estimated values and was chosen here partly from consideration of the numerical values of the dimensionless model parameters.

In order to completely determine the dimensionless parameters given by Eq. (11) Eq. (13), we must choose values for the reference length, $w$, and the interface thickness $\delta$. The choice of these parameters is based on the physical structure we wish to compute and the practical limitations of accurately resolving gradients within the interfacial region for a desired computational domain of size $X_{L}$ and $Y_{L}$. For the simulation of dendritic growth, we choose to relate $\delta$ and $w$ to an estimate of the dendrite tip radius. Clearly, we would expect that $\delta$ must be much smaller than the tip radius of the dendrite and that the domain must be many times larger than the tip radius to simulate the growth and development of the dendrite. In order to begin the calculations, we estimated the dendrite tip radius based on marginal stability theory [14] for a given value of undercooling, represented nondimensionally here by $\Delta$. The computations are facilitated by lower values of $\Delta$ (large values of undercooling, $\Delta T$ ), because the dendrite grows faster and encompasses a larger portion of the computational domain. Values of $\Delta$ in the range of $0.4-0.5$ are used here. A dimensionless undercooling of $\Delta=0.5$ corresponds to an actual undercooling of $217 \mathrm{~K}$ for nickel, which is an attainable level of undercooling [20]. For an undercooling corresponding to $\Delta=0.5$, marginal stability gives an estimated value for the tip radius of $1.7 \times 10^{-5} \mathrm{~cm}$. Based on some preliminary computational experiments to determine 
how the interface thickness and the size of the domain should be chosen in relation to the tip radius, we obtained the parameter value $\alpha=400$ in the definition Eq. (11). For the physical parameters of nickel given above, this yields a value for the length scale $w=2.1 \times 10^{-4} \mathrm{~cm}$; the dimensionless parameter $m$ has the value 0.05 for nickel.

With the parameters $\alpha$ and $m$ specified, the parameter $\tilde{\epsilon}$ was used in the computations to vary the thickness of the interface. The computational resolution was determined by the extent of the domain chosen $\left(X_{L}\right.$ and $\left.Y_{L}\right)$ and the number of grid points used in the discretization of the domain. We performed computations with $\tilde{\epsilon}$ equal to $0.005,0.0033$, and 0.0025 , but for the majority of the simulations of dendritic growth into an undercooled liquid we used the value $\tilde{\epsilon}=0.005$.

\subsection{Numerical Method}

One of the clear advantages of the phase field model is that the location of the solid/liquid interface does not have to be determined explicitly. However, accurate numerical solutions to the phase field equations require that gradients of the field variables be adequately resolved over the thin interfacial region. Our objective is to evaluate the behavior of the phase field model presented here by simulating the growth of a two-dimensional dendrite into an undercooled liquid. In order to perform the simulation, the governing equations are solved numerically in a two-dimensional rectangular domain of sufficient size to allow for the development of characteristic dendritic structure (e.g., side arms). Even with the phase field approach, an optimum solution procedure should employ some type of adaptive technique, particularly for the phase variable, since the solution varies over such a small part of the domain. Since our interest is primarily to evaluate the model itself, we have chosen to use straightforward finite difference solution techniques applied on a uniform computational grid. An advantage of this approach is that the numerical implementation of such techniques is quite simple and it is easy to take full advantage of highly vectorized large-scale computers.

The governing equations given by Eq. (7) and Eq. (23) are a pair of coupled, second- 
order, nonlinear parabolic equations. They are discretized spatially using second-order finite differences on a uniform grid characterized by mesh spacings $\Delta X$ and $\Delta Y$ in the $x$ and $y$ coordinate directions, respectively; for the temporal discretization we introduce the time step $\Delta t$. In order to maximize the computational efficiency, we employ explicit time-differencing on the $\phi$ equation which is nonlinear in all terms except for the highestorder spatial derivatives. The heat Eq. (7) is linear in the temperature $u$ but contains the source term depending on $\phi$. With explicit time-differencing Eq. (7) would be subject to a more restrictive time step requirement than Eq. (23); thus, we employ the alternatingdirection implicit method (ADI) on Eq. (7) which for the simple linear heat equation is unconditionally stable and second-order accurate in space and time. The methods employed here are described in many standard texts on finite difference techniques for partial differential equations (for example, Richtmyer and Morton [29]).

For the nonlinear phase field equation with explicit time-differencing (e.g., simple Euler, Adams-Bashforth), the unsteady linear diffusion part of the equation is subject to the stability restriction: $\Delta t \leq(\Delta X)^{2} /(4 m)$; however, the additional nonlinear terms in the equation may impose a more restrictive condition on the size of the allowable time step. The actual size of the time step was determined by numerical experimentation. Both firstand second-order accurate explicit time differencing were evaluated for solving Eq. (23); however, the first-order method was used to obtain the results presented here, since the restriction on the size of the time step for the $\phi$ equation was such that first-order accuracy in time proved to be adequate to obtain a good balance in the spatial and temporal truncation error.

\subsection{Computation of Dendrites}

Using the numerical technique described above we have sought to compute the evolution of dendritic structures using the parameter values $m=0.05$ and $\alpha=400$ which were determined from the physical properties for nickel as described previously. The dimensionless parameters $\bar{\epsilon}, \Delta$, and $\gamma$ are varied in the calculations as is the size of the computational 
domain. We assume 4-fold anisotropy for all the calculations presented here so the mode number $k$ has the value 4 .

We found that in the absence of anisotropy $(\gamma=0)$ the solid developed as an amorphous structure in which the dendrite tips were subject to repeated tip splitting. A typical example of this behavior is shown in Figure 3(a). For nonzero values of the anisotropy parameter $\gamma$, a very distinctive needle crystal formed along each coordinate direction as a result of the four-fold symmetry of the anisotropy as shown in Figure 3(b). This is consistent with the recent microscopic solvability theory of dendrite growth, which indicates the importance of surface energy anisotropy, as well as calculations on local models of solidification [15].

For smaller values of the anisotropy ( $\gamma$ less than approximately 0.01 ), the tip did not settle down to a steady state over the period of the computation; it is not clear from our calculations whether a steady state would have occurred on a larger computational domain over a longer period of dimensionless time. However it is clear that anisotropy has a profound effect on the evolution of the crystal. For values of the anisotropy parameter $10^{-2} \leq \gamma \leq 2 \times 10^{-2}$ the dendrite tip rapidly locked into a definite steady operating state for the computational domains we employed. Unless otherwise stated all computations presented below for quantitative comparison with non-zero anisotropy yielded a needle crystal.

In order to quantitatively describe dendritic growth we compute the the tip temperature $\bar{U}$, radius $\bar{R}$, and velocity $\bar{V}$ (overbars denote phase field results) for the dendrite aligned with the $\mathrm{x}$-axis in the following manner: we define the interface by the locus $\phi(x, y, t)=1 / 2$ and so estimate the the dendrite tip position on the $\mathrm{x}$-axis, and the corresponding temperature and curvature, at each time level, by linear interpolation from the mesh. The radius of curvature of the tip was approximated at each mesh point on the $\mathrm{x}$-axis by employing the identity

$$
\frac{1}{R}=\frac{\phi_{y y}}{\phi_{x}}
$$

where second-order accurate finite differences were used to approximate the partial deriva- 

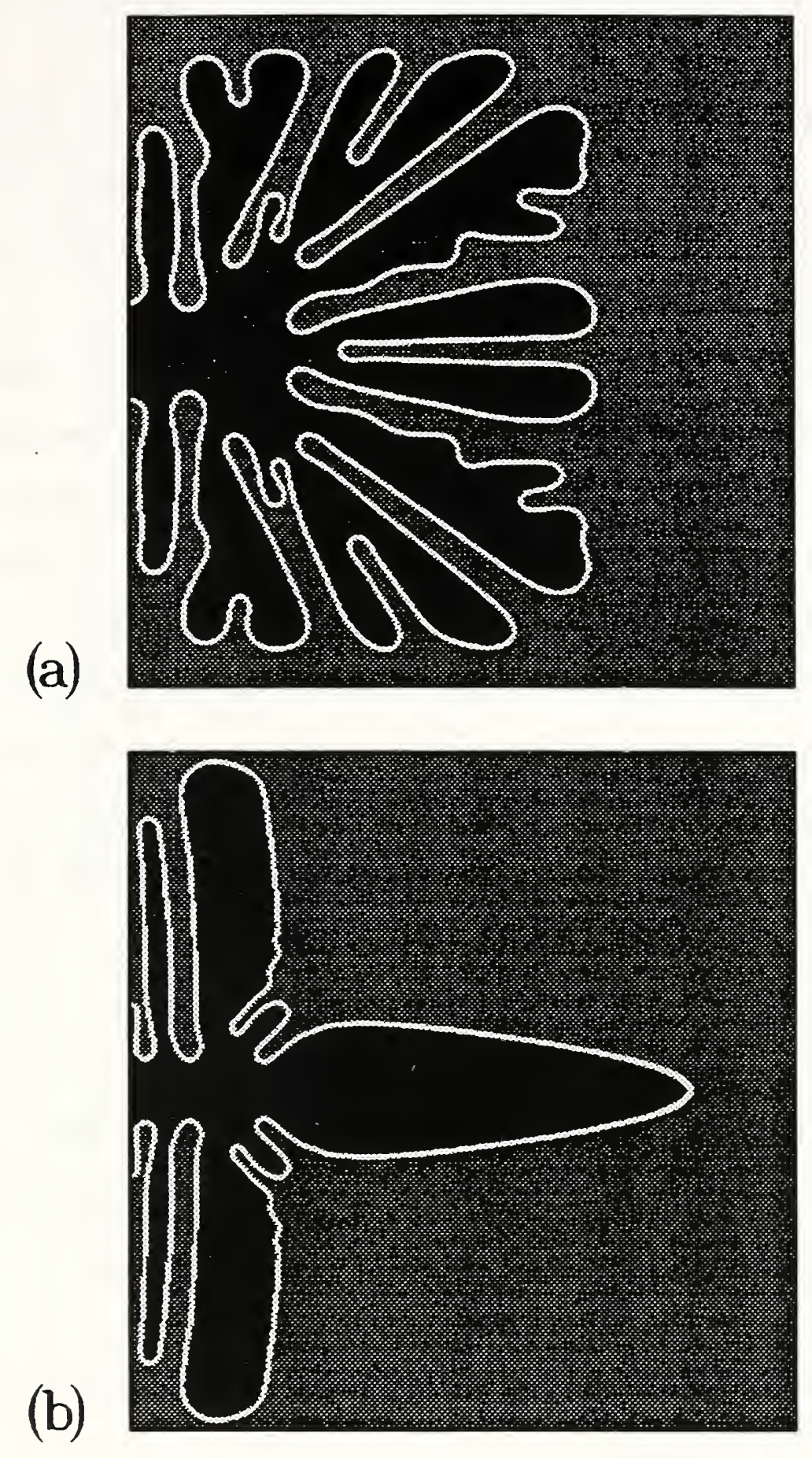

Figure 3: The computed phase field for parameters corresponding to nickel with $\bar{\epsilon}=0.005$, $\Delta=0.5, X_{L}=4.5$ and $Y_{L}=2.25$; note that the computational domain has been reflected about the horizontal centerline. The results show the the effect of anisotropy level:

(a) $\gamma=0$ and (b) $\gamma=0.01$. The black area is solid $0 \leq \phi \leq 0.1$; the white area is the interfacial region $0.1 \leq \phi \leq 0.9$; the gray area is liquid $0.9 \leq \phi \leq 1$. 
tives. The tip velocity was approximated at the the midpoint of each time interval by using central differences on the dendrite tip position.

In Figure 4 we plot $\bar{V}, \bar{R}$ and $\bar{U}$ as a function of time. There is clearly a very welldefined operating state for the tip over the greater part of the time interval, following an initial transient as the tip quickly attains its operating state. The final transient is due to the dendrite tip encountering the end of the box.

In performing the numerical computations several issues had to be addressed in order to evaluate the behavior of the present phase field model. First, we consider the effect of spatial mesh size and time step for a fixed set of dimensionless parameters. In Table 2 , we list computed values of the interface velocity and dendrite tip radius for different mesh spacings and time steps. We note here that the mesh spacing in each direction, $\Delta X$ and $\Delta Y$, are always taken to be equal to one another in all the calculations. For the finer spatial meshes, the time step restriction of the explicit treatment of the $\phi$ equation only permitted stable numerical solutions for the smaller time step values. It is apparent from the results that the tip velocity is more sensitive to the discretization error than the tip radius. We note that the computation time for $\Delta X=0.0025$ and $\Delta t=2.5 \times 10^{-5}$ is 16 times greater than for the case with $\Delta X=0.005$ and $\Delta t=1 \times 10^{-4}$, but with only a corresponding change of about $10 \%$ in the numerical values. In light of this, for the calculation of dendrites presented below, we employ the coarser of these two meshes and the larger of the time steps in order to adequately investigate the parameter space. In the calculations presented here, we estimate that there are approximately nine spatial mesh points within the interfacial region when $\Delta X=\bar{\epsilon}$; this level of resolution was used unless otherwise stated. We note that in a similar computation, Kobayashi [2] employed a mesh approximately six times coarser.

We now discuss the effect of the interface thickness on the computed results. For the parameter values corresponding to nickel (i.e., $\alpha=400$ and $m=0.05$ ), an interface thickness (defined to be represented by $\phi$ values between 0.1 and 0.9 ) corresponding to $\bar{\epsilon}=$ 0.005 was found to be the largest allowable; for $\bar{\epsilon}$ equal to 0.01 , the interfacial region is spread apart to such an extent that it no longer approximates a thin interface, while for 

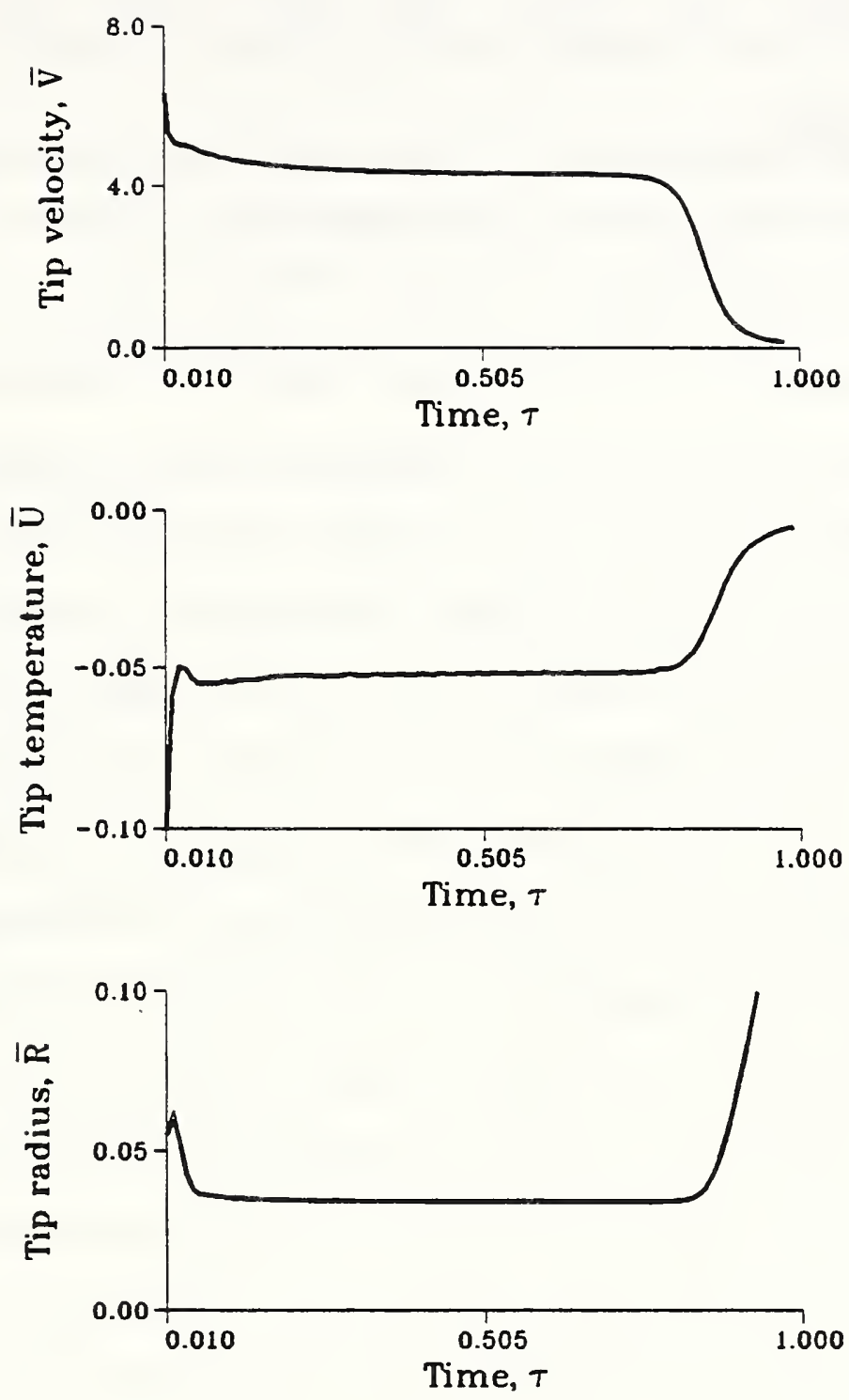

Figure 4: The estimated values of the dimensionless dendrite tip velocity $\bar{V}$, temperature $\bar{U}$, and radius $\bar{R}$, against time $\tau$ for the case $\bar{\epsilon}=0.005, \gamma=0.015$, and $\Delta=0.4$. 


\begin{tabular}{||c||cc|cc|cc|cc||}
\hline \hline \multicolumn{1}{|c||}{$\Delta t$} & \multicolumn{2}{c|}{$\Delta X=0.01$} & \multicolumn{2}{c|}{$\Delta X=0.005$} & \multicolumn{2}{c|}{$\Delta X=0.003$} & \multicolumn{2}{|c|}{$\Delta X=0.0025$} \\
$\Delta t$ & $\bar{V}$ & $\bar{R}\left(10^{2}\right)$ & $\bar{V}$ & $\bar{R}\left(10^{2}\right)$ & $\bar{V}$ & $\bar{R}\left(10^{2}\right)$ & $\bar{V}$ & $\bar{R}\left(10^{2}\right)$ \\
\hline $1.0 \times 10^{-4}$ & 4.57 & 7.34 & 5.43 & 6.79 & -- & -- & -- & -- \\
$5.0 \times 10^{-5}$ & 4.70 & 7.14 & 5.62 & 6.76 & 5.85 & 6.65 & -- & -- \\
$2.5 \times 10^{-5}$ & 4.84 & 7.11 & 5.72 & 6.74 & 5.96 & 6.61 & 6.04 & 6.53 \\
\hline \hline
\end{tabular}

Table 2: The calculated tip velocity $\bar{V}$ and tip radius $\bar{R}$ for different mesh spacings and time steps. The results were computed for parameters $\bar{\epsilon}=0.005, \gamma=0.01, \Delta=0.5$, $\alpha=400$, and $m=0.05$ on a domain with $X_{L}=2.0$ and $Y_{L}=1.0$.

$\bar{\epsilon}$ equal to 0.005 or smaller the interfacial region remains thin. We conducted a series of calculations in which only the interface thickness was varied. In Table 3 , we show the dependence of the dimensionless tip velocity, radius, and temperature on the interfacial thickness. We also list the percentage difference between the computed tip temperature and that predicted by the modified Gibbs-Thomson condition Eq. (16) using the computed tip velocity and radius; this provides a quantitative measure of the accuracy of the phase field approximation to the sharp interface model Eq. (14), Eq. (15) and Eq. (24). It is evident that the operating state of the dendrite is sensitive to the interface thickness. However, as the interface width is reduced the error in the modified Gibbs-Thomson equation is diminished. The interface moves more slowly at thinner interface widths. Similar behavior was observed in the spherically symmetric calculations discussed Section 3.3. It is clear that in order to employ the phase field method as an accurate computational approach for approximating the solution to the sharp interface model, sufficiently thin interfaces must be taken which must be adequately resolved by the computational mesh. This we believe is the major computational issue to be addressed in the subsequent development of the phase field method as an accurate computational technique.

From the above discussion in order to adequately resolve the interfacial layers we require $\Delta X=\bar{\epsilon}$, which for a value of $\bar{\epsilon}$ of $2.5 \times 10^{-3}$ produces the smallest error obtained in the phase field approximation. However, for the finite difference algorithm with a uniform spatial mesh this requires an impractical amount of computing resources for the 


\begin{tabular}{||c||c|c|c|c||}
\hline \hline $\bar{\epsilon}$ & $\bar{V}$ & $\bar{R}\left(10^{2}\right)$ & $\bar{U}\left(10^{2}\right)$ & $\frac{\left(\widetilde{\left.U_{G T}-\tilde{U}\right)}\right.}{U} \times 100$ \\
\hline $5.0 \times 10^{-3}$ & 5.43 & 6.79 & -3.86 & $82 \%$ \\
$3.3 \times 10^{-3}$ & 3.89 & 7.94 & -4.32 & $19 \%$ \\
$2.5 \times 10^{-3}$ & 3.32 & 10.23 & -3.90 & $11 \%$ \\
\hline \hline
\end{tabular}

Table 3: The calculated tip velocity $\bar{V}$, tip radius $\bar{R}$, and interface temperature $\bar{U}$ for three values of the interface thickness. The last column is the percentage difference of the calculated tip temperature to the temperature $\bar{U}_{G T}$ computed from the modified GibbsThomson Eq. (24) using the $\bar{V}$ and $\bar{R}$ values. The results were computed for parameters $\gamma=0.01, \Delta=0.5, \alpha=400$, and $m=0.05$ on a domain with $X_{L}=2.0$ and $Y_{L}=1.0$; in each case the mesh spacing was given by $\Delta X=\bar{\epsilon}$.

simulation of dendrite growth. The differences given in Table 3 represent a worst case and we found that with $\bar{\epsilon}=5.0 \times 10^{-3}$ the error in satisfying the modified Gibbs-Thomson equation was typically $25 \%$. We go on to describe results of dendritic growth which must be regarded in this context. Our aim is to further investigate the phase field model, and, in particular, the relation of the computed dendritic solutions to existing theories of dendrites, with which we find surprisingly good agreement.

The dimensions of the computational domain, $X_{L}$ and $Y_{L}$ were 4.5 and 2.25 , respectively. Initially the solid region was an ellipse with semi-major axis $x_{0}=0.5$ and semiminor axis $y_{0}=0.05$; both the solid and liquid had a dimensionless temperature of -1 . We employed a mesh of 900 and 450 uniform intervals in the $x$ and $y$ directions respectively, corresponding to equal mesh sizes $\Delta X=\Delta Y=0.02$, with a time step $\Delta t=10^{-4}$. Simple Euler time stepping was used to advance the solution in time. This mesh was the finest we could practically employ consistent with doing sufficient runs to adequately cover the $(\gamma, \Delta)$ parameter space.

Our first comparison is against the Ivantsov similarity solution for a parabolic interface propagating with constant velocity in the direction of its axis of symmetry into an infinite undercooled melt. This solution assumes that the interface temperature is constant and equal to the melting point, and so interface surface energy and interface kinetic effects are neglected. It predicts in two-dimensions that the Peclet number is related to the 
dimensionless undercooling $\Delta$ as

$$
\Delta=\sqrt{\pi \mathcal{P}} \exp (\mathcal{P}) \operatorname{erfc}(\sqrt{\mathcal{P}})
$$

where $\mathcal{P}=2 v r / \kappa$ and $\Delta=c\left(T_{M}-T_{\infty}\right) / L$. Here $v$ and $r$ are the dimensional tip velocity and radius, $T_{M}$ is the melting temperature which is equal to the isothermal interface temperature, and $T_{\infty}$ is the far-field temperature. Our phase field model includes both surface energy and interface kinetic effects and so the interface is consequently not isothermal with a temperature equal to the melting temperature. This is confirmed in Figure 4 which shows that the dendrite tip temperature $\bar{U}$ is depressed beneath the melting point (given as zero in our non-dimensionalization). For purposes of comparison to the Ivantsov model, which only accounts for conservation of heat, we estimate the undercooling parameter by $\bar{\Delta}=c\left(T_{\text {tip }}-T_{\text {init }}\right) / L,(=\bar{U}+1)$, where $T_{\text {init }}$ is the initial temperature of the liquid in the phase field calculations. The initial temperature $T_{\text {init }}$ is a good approximation to $T_{\infty}$ in the Ivantsov model, because at the large undercoolings we employ the temperature gradient is well-confined to the vicinity of the dendrite during the period of constant velocity tip growth. In Figure 5 we compare the Peclet numbers estimated from our computations, $\overline{\mathcal{P}}=2 \bar{R} \bar{V}$, for different values of the dimensionless undercooling, $\bar{\Delta}$, against those predicted by the Ivantsov formula, $\mathcal{P}_{I}(\Delta)$, shown as a solid curve. Here, the function, $\mathcal{P}_{I}(\Delta)$, is obtained by inverting the Ivantsov formula Eq. (25). The value of the anisotropy parameter is $\gamma=10^{-2}$. The agreement between the two is fairly good, improving as the undercooling decreases. In Figure 6 we plot the ratio of the estimated Peclet number to that given by the Ivantsov formula against the anisotropy parameter for $\Delta=0.4$. We chose to plot the ratio $\overline{\mathcal{P}} / \mathcal{P}_{I}(\bar{\Delta})$, because the estimated undercooling parameter, and hence the Peclet number, predicted from the Ivantsov theory $\mathcal{P}_{I}(\bar{\Delta})$ varies between the calculations for different anisotropy parameters. We see from Figure 6 that the agreement to the Ivantsov theory improves as the anisotropy diminishes. It would appear that extrapolation of the almost linear dependence of $\overline{\mathcal{P}} / \mathcal{P}_{I}(\bar{\Delta})$ to $\gamma=0$ would reveal that the Ivantsov solution would not be recovered for zero anisotropy. However, as noted above no steady solution was found for $\gamma<10^{-2}$, and so it is not clear whether 


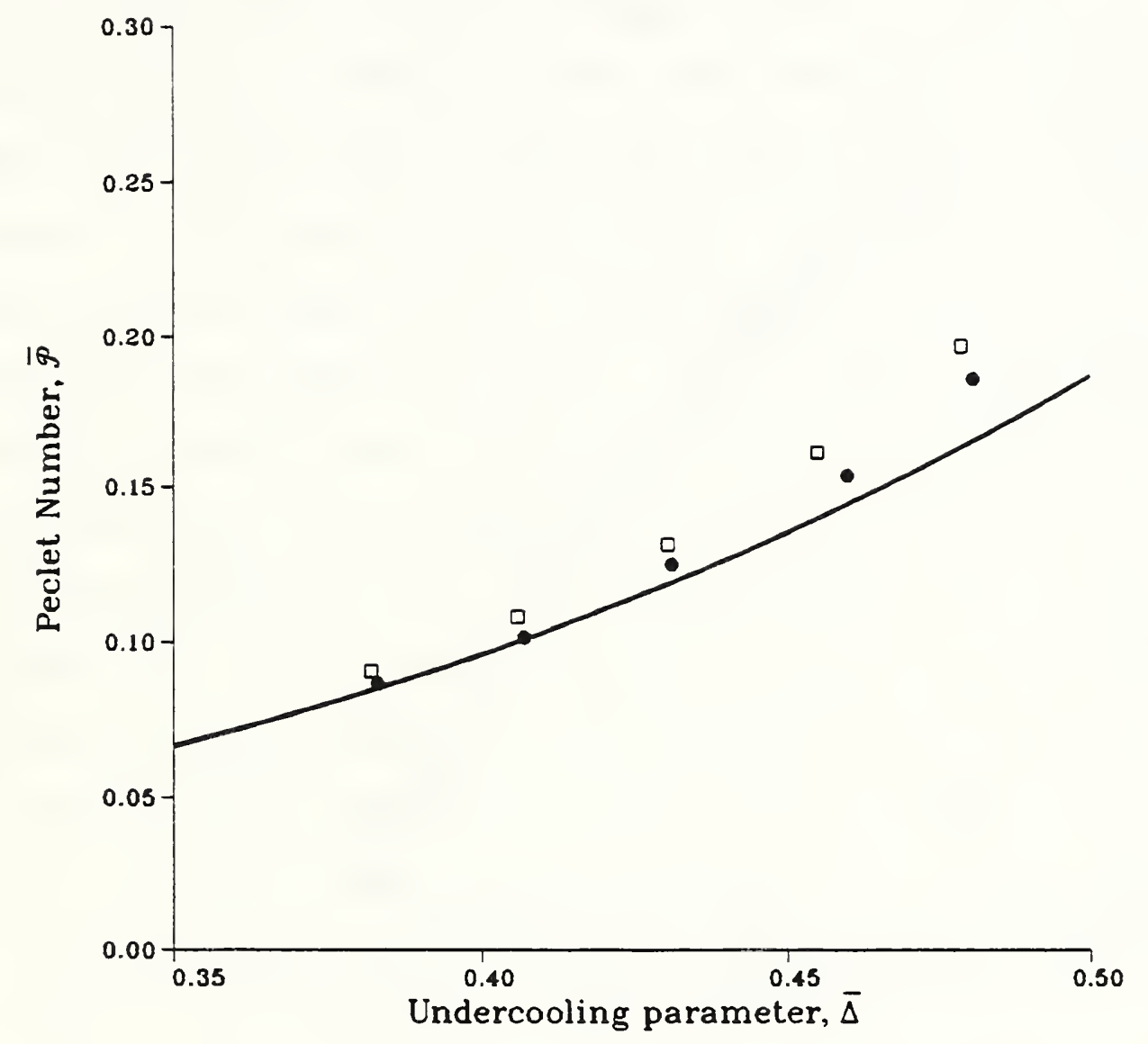

Figure 5: The Peclet number from our calculations $\overline{\mathcal{P}}$ against the estimated undercooling parameter $\bar{\Delta}$ for nickel. The solid circles represent runs on the domain $X_{L}=4.5$ and $Y_{L}=2.25$ with mesh size $5 \times 10^{-3}$; the open squares on the domain $X_{L}=2.0$ and $Y_{L}=1.0$ with mesh size $2.5 \times 10^{-3}$. The solid curve is the prediction from the Ivantsov formula Eq. (25). 


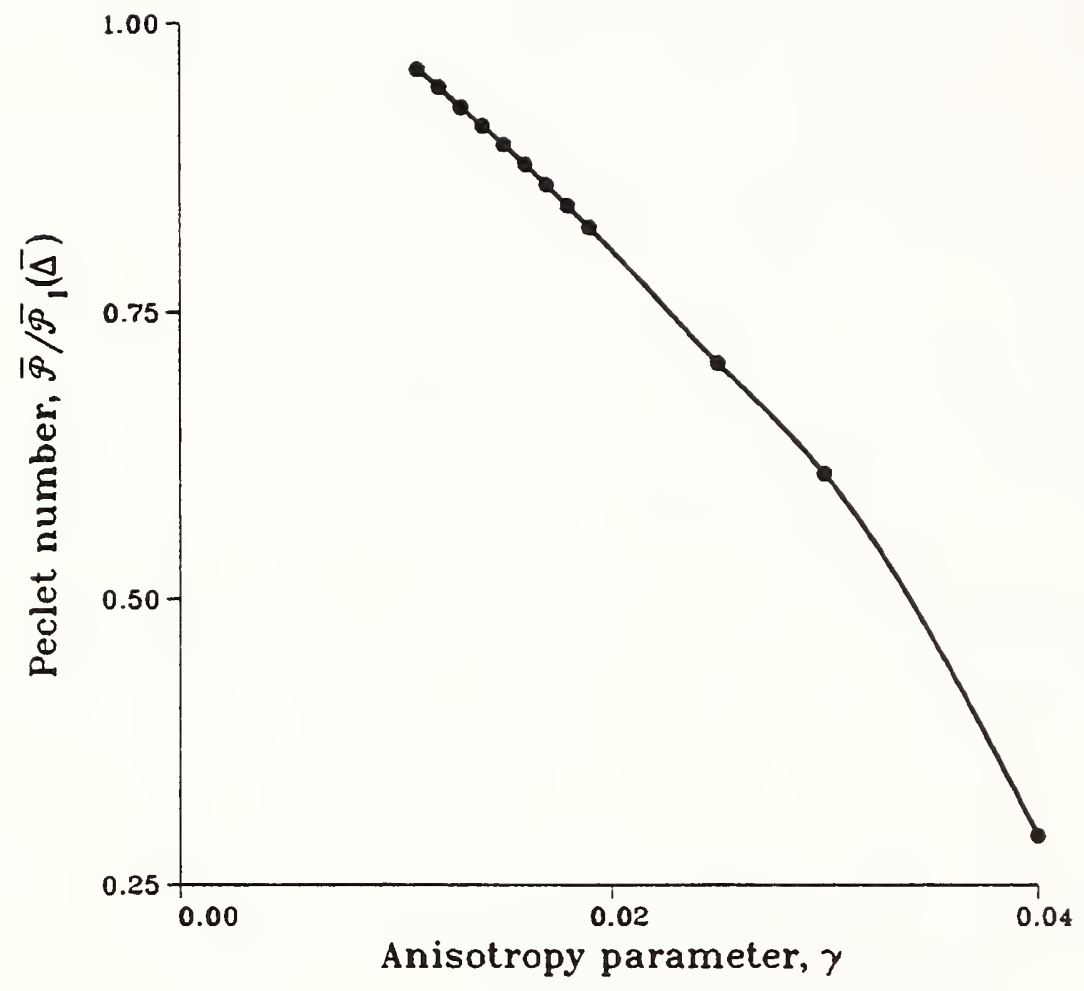

Figure 6: The ratio of the estimated Peclet number $\overline{\mathcal{P}}$ to that predicted by the Ivantsov solution $\mathcal{P}_{I}(\bar{\Delta})$ plotted against the anisotropy parameter $\gamma$ with undercooling parameter $\bar{\Delta}=0.4$ 
there is any justification for such an extrapolation. In Figure 7 we compare the results of our computations to marginal stability theory. Marginal stability theory predicts that

$$
\sigma^{\star}=\frac{2 \sigma}{L v r^{2}}=0.0192 .
$$

In this figure we plot the values of $\bar{\sigma}^{\star}$ from our computations against the dimensionless undercooling parameter $\bar{\Delta}$ for the smallest value of the anisotropy parameter $\left(\gamma=10^{-2}\right)$ for which we obtained a well defined steady dendrite tip operating state. The solid horizontal line indicates the marginal stability result given above. The results show increasing agreement with the marginal stability result with decreasing undercooling. The dependence of $\bar{\sigma}^{\star}$ on $\bar{\Delta}$ is unexpected, and may be due to the inclusion of interface kinetics in the phase-field model, which we expect to be significant at the large values of the dimensionless undercooling used in the calculations, but which are absent in both marginal stability and microscopic solvability theory. In Figure 8 we display $\bar{\sigma}^{\star}$ against the anisotropy parameter $\gamma$ for a fixed value of $\Delta$. The dashed line indicates the best power law fit through the data which corresponds to $\sigma^{\star} \propto \gamma^{1.896}$. Microscopic solvability theory predicts the exponent in the power law to be 1.75. The data points for values of $\gamma$ in excess of $2 \times 10^{-2}$ consist of dendrites whose tip radius $(\bar{R}<0.02)$ is well less than the interface thickness $(\approx 0.2)$ and therefore we may expect these results to be less reliable. Also shown in Figure 8 as solid squares are the results of microscopic solvability theory given by Ben Amar [19] who numerically computed the value of $\sigma^{\star}$. The level of agreement is surprisingly good, particularly in view of the fact the we are conducting computations on a finite domain using the phase field model with a relatively large value of the interface width which, as we have discussed above, is not a particularly good approximation to the classical free boundary problem obtained as $\tilde{\epsilon} \rightarrow 0$. Moreover, this free boundary problem includes interface kinetics which is absent in the microscopic solvability theory. In view of these limitations, the estimated exponent provides some evidence to suggest that microscopic solvability theory and the phase field model in two dimensions contain a measure of agreement. The experiments conducted by Willnecker et al. [20] on the solidification of nickel measured the dendrite tip velocity to be in the range 37 to $48 \mathrm{~ms}^{-1}$, our calculations 


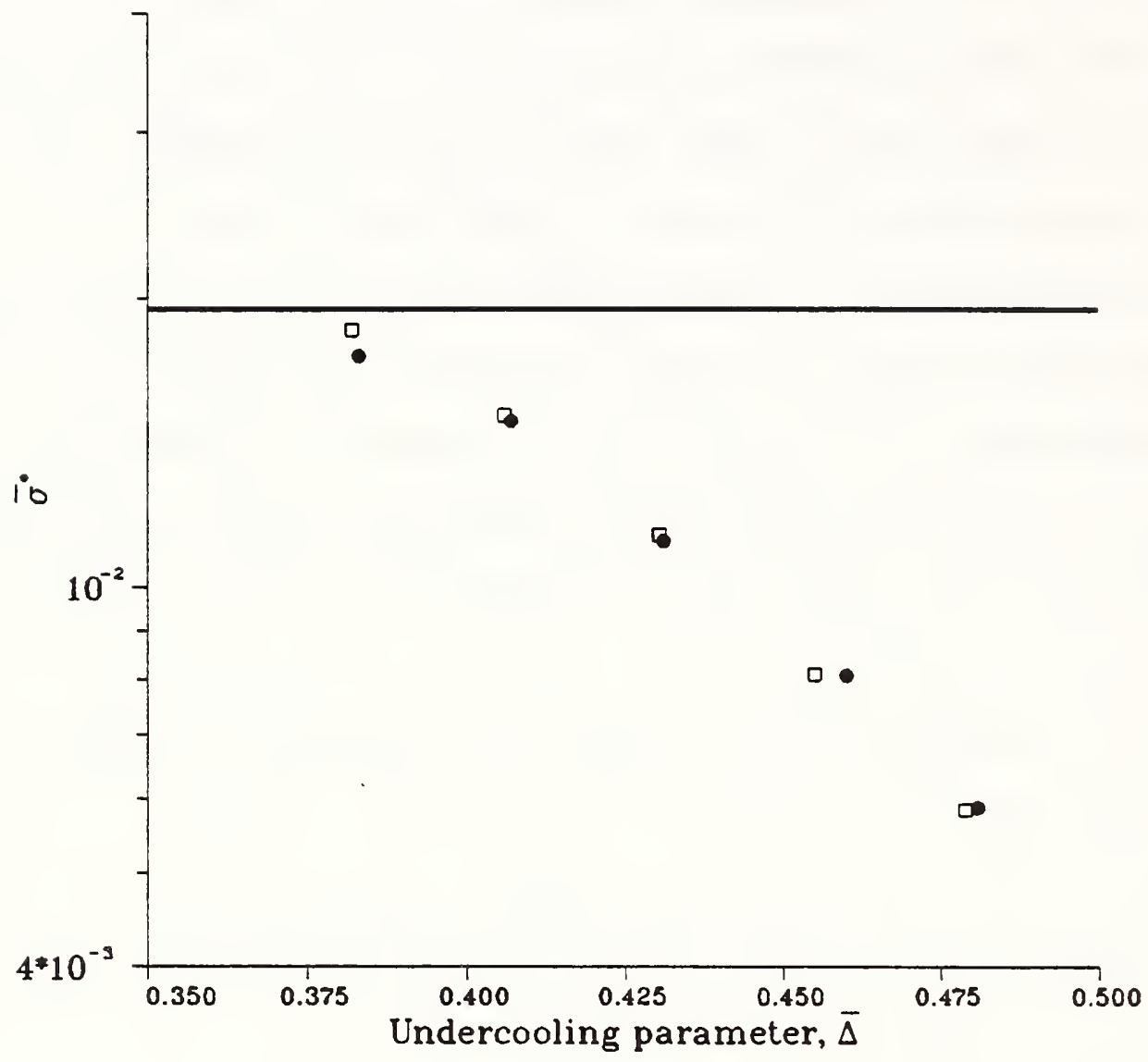

Figure 7: The calculated value of $\bar{\sigma}^{\star}$ plotted against the undercooling parameter $\bar{\Delta}$. The solid circles represent calculations on the domain $X_{L}=4.5$ and $Y_{L}=2.25$ with mesh size $5 \times 10^{-3}$; the open squares where computed on the domain $X_{L}=2.0$ and $Y_{L}=1.0$ with mesh size $2.5 \times 10^{-3}$. The horizontal line corresponds to the value $\sigma^{\star}=0.0192$ from marginal stability theory. 


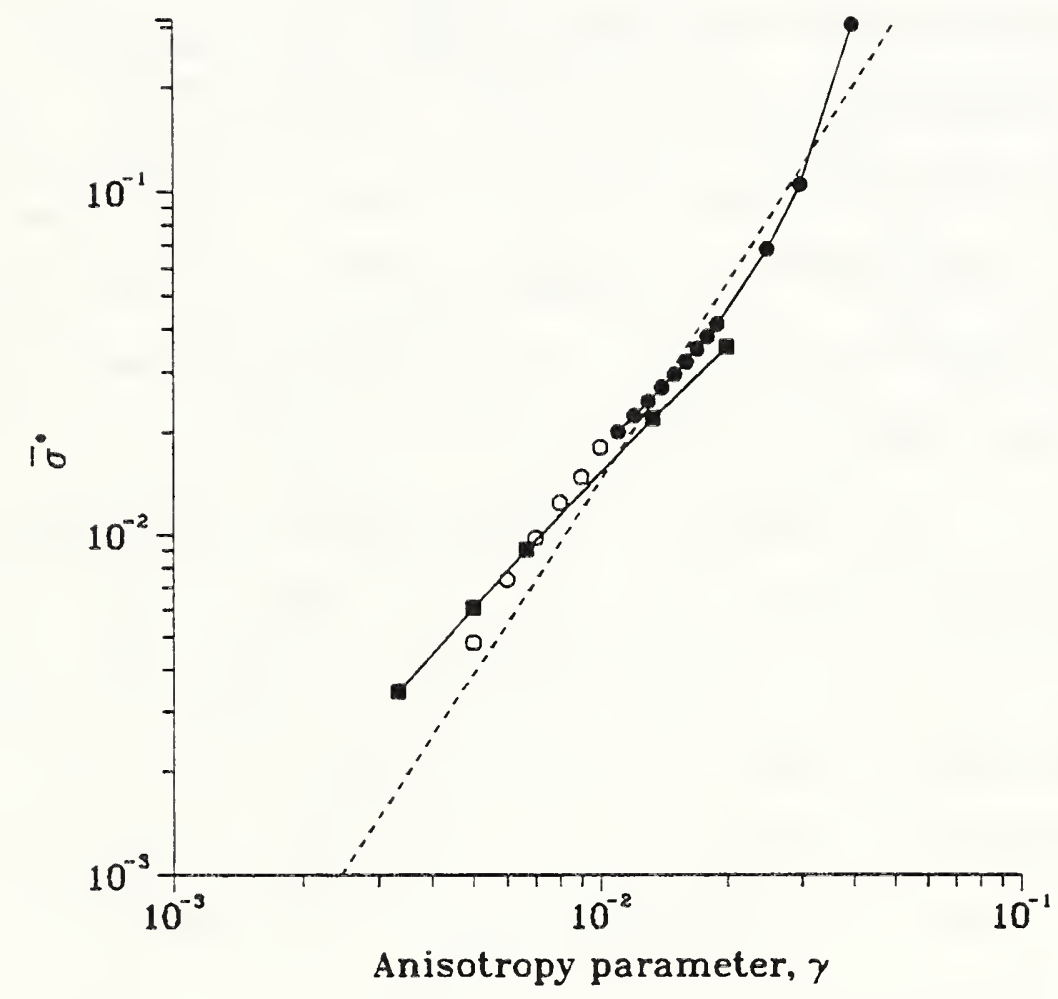

Figure 8: The calculated value of $\bar{\sigma}^{\star}$ plotted against the anisotropy parameter, $\gamma$, with undercooling parameter $\Delta=0.4$. The results were computed on the domain $X_{L}=4.5$ and $Y_{L}=2.25$ with mesh size $5 \times 10^{-3}$; the open circles represent calculations for which a steady operating state was not obtained. The solid squares correspond to values of $\sigma^{\star}$ computed by Ben Amar [19] on the basis of microscopic solvability theory. The dashed line is the power law fit to the solid circles. 
predict tip velocities in the range 26 to $50 \mathrm{~ms}^{-1}$ for the anisotropy parameter in the range 0.011 to 0.04 . The value of $\gamma$ for most materials, including nickel, is difficult to measure and unknown. However, despite this uncertainty in the value of $\gamma$ our calculations reveal growth rates in the correct range, although we note that the experiments measured three dimensional dendrites compared to our two dimensional calculations.

Finally, we use the present phase field model to simulate the development of a sidebranched dendritic structure. We found that the formation of side-branches was dependent on the spatial resolution used. For spatially underresolved calculations, with approximately 3-5 mesh points within the interfacial layer, uniformly spaced side-branches evolved. However, when the spatial resolution was increased the side-branching disappeared. We attribute the formation of side-branched structure on coarser meshes to the noise associated with the larger truncation error. To investigate this further, we introduced random noise into the phase field equation for computations on finer meshes. In particular, we added the term $A_{n} r_{n}$ into the expression in square brackets in the discretized form of Eq. (23), where $A_{n}$ is the amplitude of the noise and $r_{n}$ is a random number in the interval $[-0.5,0.5]$. Physically, this represents thermal noise at the interface and was also used by Kobayashi [1]. The production of side arms was very sensitive to the noise; side arms were stimulated for values of $A_{n}$ as low as $2.5 \times 10^{-3}$. In contrast the operating state of the tip was insensitive to noise, the time average of the tip velocity and radius being only very weakly affected. This provides some evidence to suggest that side arm formation is a process distinct from the dynamics of the dendrite tip.

In Figure 9 we show a computation that displays side-branch formation. The phase field and isotherms are shown at three different dimensionless times. In the phase field plot, the width of the curve represents the interval $0.1 \leq \phi \leq 0.9$, and thus gives an indication of the thickness of the interface. For this computation, we again employed the parameter values corresponding to nickel used throughout this study. For the simulation the domain has dimensions $X_{L}=6.75$ and $Y_{L}=3.375$. The mesh spacing was $\Delta X=$ $\Delta Y=0.075$, and the time step was $\Delta t=5.0 \times 10^{-5}$. The computation starts from an elliptical solid region with semi-major axis $x_{0}=0.15$ and semi-minor axis $y_{0}=0.075$. The 
dimensionless parameters are $\bar{\epsilon}=0.005, \gamma=0.01$, and $\Delta=0.5$.

\section{Conclusions}

We have conducted an extensive detailed study of the phase field method as a computational procedure for the solution of free boundary problems associated with solidification, and in particular dendritic interfaces. On the basis of the simple finite difference methods employed here we find that in simple one dimensional geometries accurate solutions can be obtained. However, in more realistic two dimensional geometries accurate computations require interfaces so thin that the need to accurately resolve them require computing resources at the limit of current computer technology. However, less accurate computations on thicker interfaces provides surprisingly good quantitative agreement with both the Ivantsov and microscopic solvability theories of dendrite tip growth. Side branch formation in these computations is caused by the introduction of very small amplitude noise, which leaves the operating state of the dendrite tip largely unaffected.

Because of the need to use very fine interface thicknesses we believe that further use of the phase field model as an accurate computational tool for the computation of two and three dimensional solid shapes will require more sophisticated numerical algorithms, possibly employing adaptive finite element techniques.

\section{Acknowledgments}

We wish to acknowledge helpful discussions with W.J. Boettinger, S.R. Coriell, G.B. McFadden, and R.F. Sekerka.

This work was conducted with the support of the Microgravity Science and Applications Division of the National Aeronautics and Space Administration and the Applied and Computational Mathematics Program of the Defense Advanced Research Projects Agency. One of the authors (AAW), gratefully acknowledges partial support from a NATO collaborative research grant. 

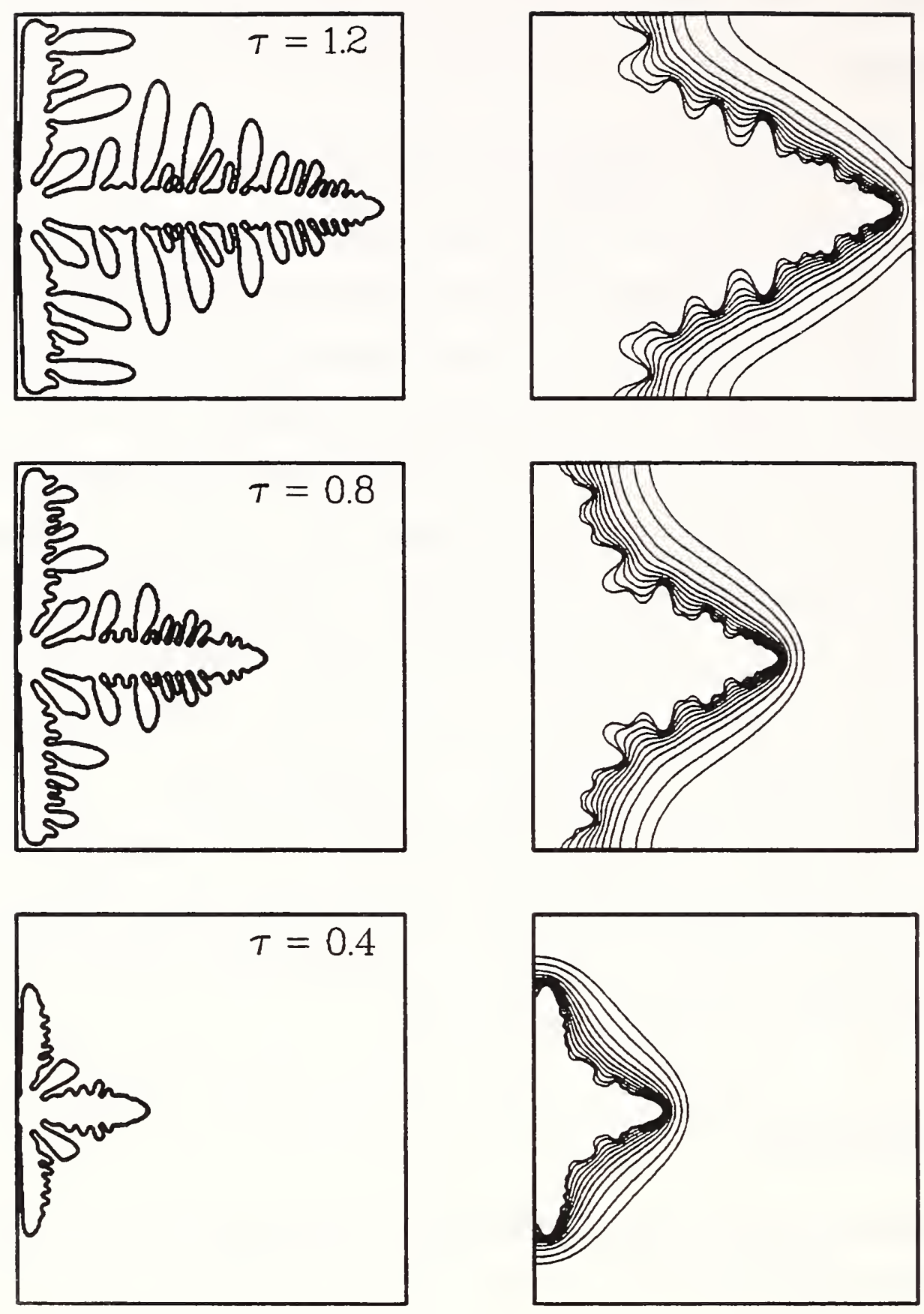

Figure 9: A simulation of a nickel dendrite with the phase field (on the left) and the temperature field (on the right) shown at three different dimensionless times $(\tau)$. The dimensionless parameters are $\bar{\epsilon}=0.005, \gamma=0.01$, and $\Delta=0.5$. Random noise with a one-percent amplitude was introduced into the calculation to stimulate the development of the side-branched structure. The computational domain was reflected about the horizontal centerline. 


\section{References}

[1] R. Kobayashi, 1991, Video tape.

[2] R. Kobayashi, Bull. Jpn. Soc. Ind. Appl. Math. 1, 22 (1991).

[3] R. Kobayashi, In press. Physica D (1992).

[4] J. S. Langer, in Directions in Condensed Matter Physics, edited by G. Grinstein and G. Mazenko, pages 164-186, World Science Publishers, 1986.

[5] B. I. Halperin, P. C. Hohenburg, and S.-K. Ma, Phys. Rev. B 10, 139 (1974).

[6] J. B. Collins and H. Levine, Phys. Rev. B 31, 6119 (1985).

[7] G. Caginalp, Arch. Rat. Mech. Anal. 92, 205 (1986).

[8] G. Caginalp, Phys. Rev. A 39, 5887 (1989).

[9] J. Smith, J. Comp. Phys. 39, 112 (1981).

[10] G. Caginalp and E. A. Socolovsky, J. Comp. Phys. 95, 85 (1991).

[11] H. Lowen, J. Bechoefer, and L. S. Tuckerman, Phy. Rev. A 45, 2399 (1992).

[12] M. E. Glicksman, R. J. Schaefer, and J. D. Ayers, Met. Trans. A. 7, 1747 (1976).

[13] G. P. Ivantsov, Dokl. Akad. Nauk. SSSR 58, 567 (1947).

[14] W. Oldfield, Mater. Sci. Engng 11, 211 (1973).

[15] D. A. Kessler, J. Koplik, and H. Levine, Advances in Physics 37, 255 (1988).

[16] Y. Pomeau and Ben Amar, in Solids Far From Equilibrium, edited by C. Godreche, chapter 4, Cambridge University Press, Cambridge, 1992.

[17] D. Meiron, Phys. Rev. A. 33, 2704 (1986). 
[18] Y. Saito, G. Goldbeck-Wood, and H. Muller-Krumbhaar, Phys. Rev. A. 38, 2148 (1988).

[19] M. Ben Amar, Phys. Rev. A. 41, 2080 (1990).

[20] R. Willnecker, D. M. Herlach, and B. Feuerbacher, Phys. Rev. Lett. 62, 2707 (1989).

[21] E. R. Rubinstein and M. E. Glicksman, J. Crystal Growth 112, 84 (1991).

[22] E. R. Rubinstein and M. E. Glicksman, J. Crystal Growth 112, 97 (1991).

[23] R. J. Schaefer and M. E. Glicksman, J. Crystal Growth 5, 44 (1969).

[24] O. Penrose and P. C. Fife, Physica D 43, 44 (1990).

[25] S. R. Coriell, G. B. McFadden, B. T. Murray, R. F. Sekerka, S-L Wang and A. A. Wheeler, (1992), In preparation.

[26] S. Osher, (1991), Private communication.

[27] R. J. Braun, S. R. Coriell, G. B. McFadden, R. F. Sekerka, A. A. Wheeler, (1992), In preparation.

[28] A. A. Wheeler, W. J. Boettinger, and G. B. McFadden, Phys. Rev. A. 45, 7424 (1992).

[29] R. D. Richtmyer and K. W. Morton, Difference Methods for Initial-Value problems, Inter-Science Publishers, John Wiley, London, England, 1957. 


\begin{tabular}{lr}
\hline NIST-114A & U.S. DEPARTMENT OF COMMERCE \\
(REV. 3-90) & NATIONAL INSTITUTE OF STANDARDS AND TECHNOLOGY
\end{tabular}

BIBLIOGRAPHIC DATA SHEET
1. PUBLCATION OR REPORT NUMBER
NISTIR 4894

2. PERFoRMING ORGANIZATION REPORT MUMBER

3. PUBLCATION DATE

\section{TITLE AND SUBTITLE}

Computation of Dendrites Using

a Phase Field Model

5. AUTHOR(S)

A. A. Wheeler, B. T. Murray, and R. J. Schaefer

6. PERFORMINO ORGANIZATION (IF JOINT OR OTHER THAN NIST, SEE INSTRUCTIONS)

U.S. DEPARTMENT OF COMMERCE

MATIONAL INSTITUTE OF STANDARDS AND TECHMOLOQY

GAITHERSBURG, MD 20899

7. CONTRACT/GRANT NUMBER

8. TYPE OF REPORT AND PERIOD COVERED

9. SPONSORING ORGANIZATION RAME AND COMPLETE ADDRESS (STREET, CTTY, STATE, ZIP)

10. SUPPLEMENTARY MOTES

11. ABSTRACT (A 200-WORD OR LESS FACTUAL SUMMARY OF MOST SIOMIFICANT INFORMATION. IF DOCUMENT INCLUDES A SIGNIFICANT BIBLOORAPHY OR ITERATURE SURVE, MENTION IT HERE.)

A phase field model is used to numerically simulate the solidifcation of a pure material. We employ it to compute growth into an undercooled liquid for a one-dimensional spherically symmetric geometry and a planar two-dimensional rectangular region. The phase field model equations are solved using finite difference techniques on a uniform mesh. For the growth of a sphere, the solutions from the phase field equations for sufficiently small interface widths are in good agreement with a numerical solution to the classical sharp interface model obtaine using a Green's function approach. In two dimensions, we simulate dendritic growth of nickel with four-fold anisotropy and investigate the effect of anisotropy level on the growth of a dendrite. The quantitative behavior of the phase field model is evaluated for varying interface thickness and spatial and temporal resolution. We find quantitatively that the results depend on the interface thickness and with the simple numerical scheme employed it is not practical to do computations with a sufficiently thin interface, such that, the numerical solution is an accurate representation of a sharp interface model. However, even with a relatively thick interface the results from the phase field model show many of the features of dentritic growth and they are in surprisingly good quantitative agreement with the Ivantsov solution and microscopic solvability theory.

12. KEY WORDS (6 TO 12 ENTRIES; ALPHABETICAL ORDER; CAPITALZE ONLY PROPER MAMES; AND SEPARATE KEY WORDS BY SEMICOLONS) dendritic growth; mathematical modeling; phase field; solidification

13. AVALABIUTY

\begin{tabular}{|l}
\hline \\
\hline
\end{tabular}

UMUMITED

FOR OFFICIAL DISTRIBUTION. DO NOT RELEASE TO MATIONAL TECHNICAL INFORMATION SERVICE (NTIS).

ORDER FROM SUPERINTENDENT OF DOCUMENTS, U.S. GOVERNMENT PRINTING OFFICE, WASHINOTON, DC 20402.

ORDER FROM MATIONAL TECHMICAL IMFORMATION SERVICE (NTIS), SPRINGFIELD, VA 22161.

14. MUMBER OF PRINTED PAGES

15. PRICE 


